\title{
A Visit to Wittig and Arsa-Wittig Reactions
}

\section{LIVIU BIRZAN*, MIHAELA CRISTEA, VICTORITA TECUCEANU, CONSTANTIN DRAGHICI, ANAMARIA HANGANU, ALEXANDRU C. RAZUS*}

Romanian Academy, Institute of Organic Chemistry "C. D. Nenitzescu”, 202B Splaiul Independentei, 060023, Bucharest, Romania

\begin{abstract}
Some considerations regarding the mechanism of arsa-Wittig condensation have been supplemented with the synthesis of compounds substituted with the nitro to phenyl and various alkyl groups to the azulene moiety. For several unknown meta-substituted 1-styrylazulenes Wittig synthesis was used. The obtained products were completely characterized. A look at the behavior of the products in NMR and UV-Vis spectrometry was taken.
\end{abstract}

Keywords: Wittig reaction, arsa-Wittig reaction, styrylazulenes, H-NMR discussion

\section{Introduction}

Among our concerns regarding the azulenes substituted with double bond [1], the synthesis and properties of 1-vinylazulenes with different substituents at the $\mathrm{C}=\mathrm{C}$ double bond represented a priority. Particular importance was given to the large classes of styrylazulenes, which was achieved by Wittig reaction [2], or by condensation of 1-azulenecarbaldehydes or Schiff bases [3] with active methylene compounds $[4,5]$.

It is known that the condensation of arsonium ylides with aldehydes, as an alternative to Wittig reaction, has often been used to obtain a large variety of alkenes. The arsa-Wittig was used because of high stereoselectivity of products and mild reaction conditions [6-8]. Experimental evidence suggests that arsonium ylides are more dipolar than their phosphonium and sulfonium analogues. At first, it was considered that arsonium ylides generate by condensation with aldehydes either alkenes or epoxides, as opposed to phosphonium or sulfonium ones, which produce only alkenes or epoxides, respectively [7]. However, other investigations of the condensation of arsonium ylides showed that, depending upon the nature of the ylide, alkene or epoxide was formed as major or unique product. The aim of this paper is to use the Wittig and arsa-Wittig condensations for the enlargement of the 1-styrylazulenes classes and to compare the two reaction routes [9].

Syntheses of 1-styrylazulenes by Wittig condensation have been published since the 1960s. Thus, 1-styrylazulene was obtained from benzyltriphenylphosphonium chloride and 1-azulenecarb-aldehyde in the presence of $n \mathrm{BuLi}$ in $\mathrm{Et}_{2} \mathrm{O}$ or from (azulen-1-ylmethyl)triphenylphosphonium iodide and benzaldehyde in the presence of the same base [10]. Briquet and Hansen, using alkaline ethoxides in boiling toluene, were able to produce several 1-styrylazulenes with unsubstituted or substituted phenyl in position 4 [11]. Despite the lower electrophilic character of the carbonyl group in position 1 of azulenes than that of other aromatic aldehydes, good yields were obtained for the condensation products starting from alkylated azulenes. The 4-dimethylamino substituent drops the yield [12] to 12 $\%$ whereas the nitro group increases the yield until $92 \%$ [1] similar to the yields obtained for the unsubstituted phenyl. The reaction produced $E$ isomer of alkene or a mixture of $E / Z$ isomers depending the starting materials.

\section{Materials and methods}

Melting points (uncorrected) were measured with Kofler apparatus (Reichert Austria). Elemental analyses were performed using a Perkin Elmer CHN 240B analyzer. UV spectra were performed using a Varian Cary 100 spectrophotometer ( $\lambda$ values are given in $\mathrm{nm}$ and the molar extinction, $\varepsilon$, in $\mathrm{M}^{-1} \mathrm{~cm}^{-1}$ units). ${ }^{1} \mathrm{H}$ and ${ }^{13} \mathrm{C}$ NMR: Bruker Advance III $500\left({ }^{1} \mathrm{H}: 500.13 \mathrm{MHz},{ }^{13} \mathrm{C}: 125.77 \mathrm{MHz}\right)$ and Varian

*email: lbirzan@yahoo.com; acrazus@yahoo.com 
Gemini $300 \mathrm{BB}\left({ }^{1} \mathrm{H}: 300 \mathrm{MHz},{ }^{13} \mathrm{C}: 75.47 \mathrm{MHz}\right.$ ), in acetone (DMSO-d 6 ). TMS was used as internal standard in $\mathrm{CDCl}_{3}$; several signals were assigned based on COSY, HETCOR and HMBC experiments. Mass spectra: Varian 1200L Triple Quadrupole LC/MS/MS spectrometer by direct injection in ESI. For column chromatography, silica gel 60 or alumina [II-III Brockmann grade, 70e230 mesh ASTM] were used. The dichloromethane (DCM) was distilled over $\mathrm{CaH}_{2}$ and the ether was stored over sodium.

\section{Wittig condensation}

To the solution of meta-substituted benzyltriphenylphosphonium salt, 1R (made according to standard procedure) [13] $(1 \mathrm{mmol})$ in $\mathrm{DCM}(4 \mathrm{~mL})$ at $-78^{\circ} \mathrm{C}$, with stirring and in inert atmosphere was added $t \mathrm{BuOK}(180 \mathrm{mg}, 1.6 \mathrm{mmol})$ when a suspension was formed. The reaction mixture was stirred for $15 \mathrm{~min}$ at low temperature and then in approx. one hour allowed to reach room temperature and at this temperature was stirred for another $15 \mathrm{~min}$. The obtained mixture was diluted with ether and benzene $(10 \mathrm{~mL}$ and $10 \mathrm{~mL})$ and washed with water two times. The organic layer was dried on $\mathrm{Na}_{2} \mathrm{SO}_{4}$, filtered and the solvent removed in vacuum. The residue was chromatographed on alumina with petroleum ether and benzene (increasing benzene ratio). Two important colored fractions were isolated: one blue, which represent the $Z$ isomer of $\mathbf{2 R}$ and the second, usually navy blue, which is the $E$ isomer. Finally, eluted the unreacted aldehydes red-violet colored.

\section{Preparation of the arsonium iodides, $3 \mathrm{X}$}

Triphenylarsine (306 mg, $1 \mathrm{mmol}$ ), benzyl chloride (1 mmol) and sodium iodide (150 mg, $1 \mathrm{mmol}$ ) were dissolved in acetonitrile $(2 \mathrm{~mL})$ and heated at reflux for $4 \mathrm{~h}$ with magnetic stirring, in inert atmosphere. The reaction mixture was let to stay at room temperature till next day, when a white precipitate was formed. Then DCM $(25 \mathrm{~mL})$ was added and gentle heated until the great majority of precipitate was dissolved remaining only a small amount of sodium chloride. The precipitate was filtered and the filtrate, cooled at room temperature, was treated with petroleum ether $(50-100 \mathrm{~mL})$ when the arsonium iodide was separated as white precipitate. After $15 \mathrm{~min}$ the precipitate was filtered and washed with little petroleum ether.

\section{Arsa-Wittig condensation in presence of sodium methoxide}

To a solution of sodium methoxide ( 2 mmols in $25 \mathrm{~mL}$ ethanol) para nitrobenzyltriphenylarsonium bromide, 4 [14] (2 mmols) was added in inert atmosphere with stirring. After 15 min, to the purple solution of formed ylide 1-azulenecarbaldehde $(1 \mathrm{mmol})$ was added with a syringe. The mixture was magnetically stirred at room temperature overnight. The alcoholic solution was diluted with water $(100 \mathrm{~mL})$ and the product was extracted with DCM $(2 \times 50 \mathrm{~mL})$. The organic extract was washed with water, dried on $\mathrm{Na}_{2} \mathrm{SO}_{4}$ and the solvent was removed. The residue was chromatographed on silica gel using at the beginning a mixture of petroleum ether and DCM and, for the final elution, only DCM. There were obtained mainly two colored fractions: first a brown one containing the styryl derivatives and the second with the unreacted aldehydes. This procedure was not effective starting from meta substituted nitrobenzyltriphenylarsonium salts. Even after 2 days at room temperature and 5-6 h of reflux, the aldehyde conversions remained very low.

\section{Arsa-Wittig condensation in presence of sodium amide}

The suspension of arsonium salt (1 mmol) and sodium amide (39 $\mathrm{mg}, 1 \mathrm{mmol})$ in benzene $(20 \mathrm{~mL})$ was treated in inert atmosphere and with stirring with 1 -azulenecarbaldehyde $(1 \mathrm{mmol})$ dissolved in a small amount of benzene. The mixture was stirred at room temperature for overnight and then refluxed for another $10 \mathrm{~h}$. The reaction mixture was diluted with benzene $(20 \mathrm{~mL})$ and washed carefully with water $(2 \times 20 \mathrm{~mL})$ (some unreacted amide can react violently with water). The organic layer was dried on $\mathrm{Na}_{2} \mathrm{SO}_{4}$ and the solvent was removed. The crude reaction mixture showed the presence of 
styrylazulene and of its corresponding epoxide however all attempts to separate individual products failed. After the chromatography on silica gel column only the unreacted aldehyde could be isolate.

\section{Product characterization}

3-Methylbenzyltriphenylphosphonium chloride, 1b. White crystals, m.p. > 300 ${ }^{\circ} \mathrm{C} .{ }^{1} \mathrm{H}-\mathrm{NMR}(500$ $\mathrm{MHz}): \delta(\mathrm{ppm})=2.08(\mathrm{Me}), 5.17\left(\mathrm{~d}, \mathrm{~J}^{\mathrm{HP}}=15.6 \mathrm{~Hz}, \mathrm{CH}_{2}\right), 6.66(\mathrm{bs}, 1 \mathrm{H}, 2-\mathrm{H}), 6.84\left(\mathrm{~d},{ }^{3} \mathrm{~J}=5.8 \mathrm{~Hz}, 1\right.$ $\mathrm{H}, 6-\mathrm{H}), 7.10\left(\mathrm{~d},{ }^{3} \mathrm{~J}=6.1 \mathrm{~Hz}, 1 \mathrm{H}, 4-\mathrm{H}\right), 7.14(\mathrm{t}, \mathrm{J}=7.7 \mathrm{~Hz}, 1 \mathrm{H}, 5-\mathrm{H}), 7.69\left(\mathrm{dd},{ }^{\mathrm{HP} J}=12.3, \mathrm{~J}=7.7, \mathrm{~Hz}\right.$, $\left.6 \mathrm{H}, 2^{\prime}-\mathrm{H}, 6{ }^{\prime}-\mathrm{H}\right), 7.74\left(\mathrm{dt}, \mathrm{J}=8.1{ }^{\mathrm{HP}} \mathrm{J}=3.6 \mathrm{~Hz}, 6 \mathrm{H}, 3^{\prime}-\mathrm{H}, 5^{\prime}-\mathrm{H}\right), 7.90\left(\mathrm{dt}, \mathrm{J}=7.3{ }^{\mathrm{HP}} \mathrm{J}=1.6 \mathrm{~Hz}, 3 \mathrm{H}\right.$, 4'-H). ${ }^{13} \mathrm{C}-\mathrm{NMR}(75 \mathrm{MHz}): \delta(\mathrm{ppm})=20.76(\mathrm{Me}), 28.15\left(\mathrm{~d},{ }^{\mathrm{CP}} \mathrm{J}=46.5 \mathrm{~Hz}, \mathrm{CH}_{2}\right), 117.9\left(\mathrm{~d},{ }^{\mathrm{CP}} \mathrm{J}=85.5\right.$ $\mathrm{Hz}, \mathrm{C} 1$ ') 127.7 (d, $\left.{ }^{\mathrm{CP}} \mathrm{J}=8.6 \mathrm{~Hz}, \mathrm{C} 1\right), 128.0\left(\mathrm{~d},{ }^{\mathrm{CP}} \mathrm{J}=6.9 \mathrm{~Hz}, \mathrm{C} 6\right), 128.7$ (d, J = 3.1 Hz, C5), 128.8 (d, $\left.{ }^{\mathrm{CP}} \mathrm{J}=5.6 \mathrm{~Hz}, \mathrm{C} 4\right), 130.1\left(\mathrm{~d}, \mathrm{~J}=12.4 \mathrm{~Hz}, \mathrm{C} 3{ }^{\prime}, \mathrm{C}^{\prime}\right.$ '), $131.2(\mathrm{~d}, \mathrm{~J}=5.5 \mathrm{~Hz}, \mathrm{C} 2), 134.1$ (d, J = 19.8 Hz, C2', C6'), 135.1 (d, J =1.8 Hz, C4'), 137.9 (d, J = 3.2 Hz, C3). MS [ESI] (m/z): 387 [M']. Calcd. for $\mathrm{C}_{26} \mathrm{H}_{24} \mathrm{PCl}$ : C, 77.51; H, 6.00; P, 7.69; Cl, 8.80. Found: C, 77.53; H, 6.01.

3-Trifluoromethylbenzyltriphenylphosphonium iodide, 1c. White crystals, m.p. $245-6{ }^{\circ} \mathrm{C} .{ }^{1} \mathrm{H}-\mathrm{NMR}$ $(500 \mathrm{MHz}): \delta(\mathrm{ppm})=5.45\left(\mathrm{~d}, \mathrm{~J}=15.4 \mathrm{~Hz}, \mathrm{CH}_{2}\right), 7.17(\mathrm{bs}, 1 \mathrm{H}, 2-\mathrm{H}), 7.42\left(\mathrm{bd},{ }^{3} \mathrm{~J}=6.1 \mathrm{~Hz}, 2 \mathrm{H}, 6-\right.$ $\mathrm{H}), 7.51(\mathrm{t}, \mathrm{J}=7.9 \mathrm{~Hz}, 1 \mathrm{H}, 5-\mathrm{H}), 7.66(\mathrm{~d}, \mathrm{~J}=7.6 \mathrm{~Hz}, 1 \mathrm{H}, 4-\mathrm{H}), 7.71\left(\mathrm{dd},{ }^{\mathrm{HP}} \mathrm{J}=12.4, \mathrm{~J}=7.5 \mathrm{~Hz}, 6 \mathrm{H}\right.$, $\left.2^{\prime}-\mathrm{H}, 6^{\prime}-\mathrm{H}\right), 7.74\left(\mathrm{dt},{ }^{3} \mathrm{~J}=7.7,{ }^{\mathrm{HP}} \mathrm{J}=3.6 \mathrm{~Hz}, 6 \mathrm{H}, 3^{\prime}-\mathrm{H}, 5^{\prime}-\mathrm{H}\right), 7.91\left(\mathrm{t},{ }^{3} \mathrm{~J}=7.3,{ }^{\mathrm{HP}} \mathrm{J}=2.1 \mathrm{~Hz}, 3 \mathrm{H}, 6^{\prime}-\right.$ $\mathrm{H}) .{ }^{13} \mathrm{C}-\mathrm{NMR}(75 \mathrm{MHz}): \delta(\mathrm{ppm})=27.81\left(\mathrm{~d}, \mathrm{~J}=58 \mathrm{~Hz}, \mathrm{CH}_{2}\right), 117.4\left(\mathrm{~d}, \mathrm{~J}=85.8 \mathrm{~Hz}, \mathrm{C} 1^{\prime}\right), 123.7(\mathrm{q}, \mathrm{J}$ $\left.=272.4 \mathrm{~Hz}, \mathrm{CF}_{3}\right), 127.7$ (bs, C2), 129.3 (d, J = 32.2 Hz, C3), 129.7 (d, J = 8.3 Hz, C1), 130.0 (s, C5), 130.2 (d, J = 13 Hz, C3', C5'), 134.1 (d, J = 10 Hz, C2', C6'), 134.8 (bs, C6), 135.3 (d, J = 3 Hz, C4').- MS [ESI] (m/z): 421 [M+ ${ }^{+}$. Calcd. for $\mathrm{C}_{26} \mathrm{H}_{21} \mathrm{PF}_{3} \mathrm{I}$ : C, 56.95; H, 3.86; F, 10.39; I, 23.14; P, 5.65. Found: C, 56.97; H, 3.88.

3-Fluorobenzyltriphenylphosphonium chloride, 1d. White crystals, m.p. $300^{\circ} \mathrm{C} .{ }^{1} \mathrm{H}-\mathrm{NMR}(500$ $\mathrm{MHz}): \delta(\mathrm{ppm})=5.20\left(\mathrm{~d},{ }^{\mathrm{HP}} \mathrm{J}=15.8 \mathrm{~Hz}, \mathrm{CH}_{2}\right), 6.75\left(\mathrm{~d},{ }^{\mathrm{HF}} \mathrm{J}=10.0 \mathrm{~Hz}, \mathrm{~J}=1.3 \mathrm{~Hz}, 1 \mathrm{H}, 2-\mathrm{H}\right), 6.86(\mathrm{~d}$, $\left.{ }^{3} \mathrm{~J}=7.6 \mathrm{~Hz}, 1 \mathrm{H}, 6-\mathrm{H}\right), 7.15\left(\mathrm{bdd},{ }^{\mathrm{HF}} \mathrm{J}=8.7, \mathrm{~J}=8.4 \mathrm{~Hz}, 1 \mathrm{H}, 4-\mathrm{H}\right), 7.30\left(\mathrm{dt},{ }^{\mathrm{HF}} \mathrm{J}=6.8, \mathrm{~J}=7.6 \mathrm{~Hz}, 1 \mathrm{H}\right.$, 5-H), $7.71\left(\mathrm{dd},{ }^{\mathrm{HP}} \mathrm{J}=12.4, \mathrm{~J}=7.5 \mathrm{~Hz}, 6 \mathrm{H}, 2^{\prime}-\mathrm{H}, 6\right.$ '-H), $7.74\left(\mathrm{dt},{ }^{3} \mathrm{~J}=7.7,{ }^{\mathrm{HF}} \mathrm{J}=3.6 \mathrm{~Hz}, 6 \mathrm{H}, 3^{\prime}-\mathrm{H}, 5^{\prime}-\right.$ $\mathrm{H}), 7.91\left(\mathrm{t},{ }^{3} \mathrm{~J}=7.3,{ }^{\mathrm{HP}} \mathrm{J}=1.2 \mathrm{~Hz}, 3 \mathrm{H}, 6{ }^{\prime}-\mathrm{H}\right) .{ }^{13} \mathrm{C}-\mathrm{NMR}(75 \mathrm{MHz}): \delta(\mathrm{ppm})=27.81\left(\mathrm{~d},{ }^{\mathrm{CP}} \mathrm{J}=47.5 \mathrm{~Hz}\right.$, $\left.\mathrm{CH}_{2}\right), 115.4\left(\mathrm{dd}, \mathrm{J}^{\mathrm{CF}}=20.8,3.6 \mathrm{~Hz}, \mathrm{C} 4\right), 117.2\left(\mathrm{dd}, \mathrm{J}^{\mathrm{CF}}=22.5,5.5 \mathrm{~Hz}, \mathrm{C} 2\right), 117.6\left(\mathrm{~d},{ }^{\mathrm{CP}} \mathrm{J}=85.8 \mathrm{~Hz}\right.$, $\mathrm{C}^{\prime}$ '), $127.0\left(\mathrm{dd}, \mathrm{J}^{\mathrm{CF}}=2.7,5.4 \mathrm{~Hz}, \mathrm{C} 6\right), 130.2\left(\mathrm{~d}, \mathrm{~J}^{\mathrm{CP}}=12.5, \mathrm{C} 3\right.$ ', C5'), $130.9\left(\mathrm{~d}, \mathrm{~J}^{\mathrm{CF}}=8.5,23.2 \mathrm{~Hz}\right.$, $\mathrm{C} 5), 130.7\left(\mathrm{t}, \mathrm{J}^{\mathrm{CF}}=8.3 \mathrm{~Hz}, \mathrm{C} 1\right), 133.3\left(\mathrm{~d}, \mathrm{~J}^{\mathrm{CF}}=19.5, \mathrm{C}^{\prime}\right.$ ', C6'), $134.8\left(\mathrm{~d}, \mathrm{~J}^{\mathrm{CF}}=9.9 \mathrm{~Hz}, \mathrm{C}^{\prime}{ }^{\prime}\right), 161.8$ $\left.\left(\mathrm{dd}, \mathrm{J}^{\mathrm{CF}}=244.8,3.6 \mathrm{~Hz}, \mathrm{C} 3\right) . \mathrm{MS}[\mathrm{ESI}](\mathrm{m} / \mathrm{z}): 371 \mathrm{MM}^{+}\right]$. Calcd. for $\mathrm{C}_{25} \mathrm{H}_{21} \mathrm{PFI}: \mathrm{C}, 60.26 ; \mathrm{H}, 4.25 ; \mathrm{F}$, 3.81; I, 25.47; P, 6.22. Found: C, 60.25; H, 4.27.

1-[(E)-2-(3-Methoxyphenyl)ethenyl]azulene, E-2a. Green crystals, m.p. $106^{\circ} \mathrm{C} . \mathrm{UV}-\mathrm{Viz}, \lambda_{\max }(\lg$ $\varepsilon)$ : 205(4.33), 221 (4.28), 254 (4.32), 305sh (4.35), 316 (4.42), 333sh (4.31), 347 (4.31), 379 (4.24), 396 (4.30), 638 (2.56) nm. ${ }^{1} \mathrm{H}-\mathrm{NMR}(500 \mathrm{MHz}): \delta(\mathrm{ppm})=3.85$ (s, $\left.3 \mathrm{H}, \mathrm{OMe}\right), 6.81$ (ddd, J = 7.5; 2.5; $1.6 \mathrm{~Hz}, 1 \mathrm{H}, 4$ '-H), 7.15 (t, J = 9.6 Hz, $1 \mathrm{H}, 5-\mathrm{H}), 7.18$ (t, J = 9.8 Hz, $1 \mathrm{H}, 7-\mathrm{H}), 7.26$ (m, $1 \mathrm{H}, 6$ '-H), 7.27 (bs, $\left.1 \mathrm{H}, 2^{\prime}-\mathrm{H}\right), 7,28$ (t, J = 7.3 Hz, $\left.1 \mathrm{H}, 5^{\prime}-\mathrm{H}\right), 7.29$ (d, J = 16.1 Hz, $\left.1 \mathrm{H}, \mathrm{CH}=\right), 7.45$ (d, J = 4.2 $\mathrm{Hz}, 1 \mathrm{H}, 3-\mathrm{H}), 7.61(\mathrm{t}, \mathrm{J}=9.8 \mathrm{~Hz}, 1 \mathrm{H}, 6-\mathrm{H}), 7.93(\mathrm{~d}, \mathrm{~J}=16.1 \mathrm{~Hz}, 1 \mathrm{H}, \mathrm{CH}=), 8.29$ (d, J = 9.2 Hz, $1 \mathrm{H}$, 4-H), $8.34(\mathrm{~d}, \mathrm{~J}=4.2 \mathrm{~Hz}, 1 \mathrm{H}, 2-\mathrm{H}), 8.71(\mathrm{~d}, \mathrm{~J}=9.8 \mathrm{~Hz}, 1 \mathrm{H}, 8-\mathrm{H}) \cdot{ }^{13} \mathrm{C}-\mathrm{NMR}(75 \mathrm{MHz}): \delta(\mathrm{ppm})=$ 55.49 (Me), 112.0 (C6'), 113.4 (C4'), 119.7 (C2'), 120.0 (C3), 121.9 (CHPh), 123.7 (C7), 124.8 (C5), 128.4 (C1), 127.3 (CHAz), 130.4 (C5'), 134.1 (C2), 134.9 (C8), 138.5 (C1'), 137.7 (C4), 139.3 (C6), 140.9 (C8a), 144.0 (C3a), 161.1 (C3'). IR: 452, 496, 573, 588, 687, 707, 729, 780, 831, 865, 921, 947, 972, 1033, 1078, 1171, 1241, 1276, 1319, 1392, 1426, 1449, 1466, 1486, 1565, 1586, 1725, 2839, 2931, 2962, $3011 \mathrm{~cm}^{-1}$. MS [GC] (m/z): $261\left[\mathrm{M}^{+}+1\right]$. Calcd. for $\mathrm{C}_{19} \mathrm{H}_{16} \mathrm{O}: \mathrm{C}, 87.66 ; \mathrm{H}, 6.19 ; \mathrm{O}, 6.15$. Found: C, 87.67; H, 6.18.

1-[(Z)-2-(3-Methoxyphenyl)ethenyl]azulene, Z-2a. Green crystals, m.p. $72^{\circ} \mathrm{C}$. UV-Viz, $\lambda_{\max }(\lg \varepsilon)$ : 205 (4.66), 256 (4.39), 309 (4.55), 387 (4.10), 614 (2.48) nm. ${ }^{1} \mathrm{H}-\mathrm{NMR}(500 \mathrm{MHz}): \delta(\mathrm{ppm})=3.62(\mathrm{~s}$, $3 \mathrm{H}, \mathrm{OMe}), 6.65(\mathrm{~d}, \mathrm{~J}=12.1 \mathrm{~Hz}, 1 \mathrm{H}, \mathrm{CH}=), 6.78\left(\mathrm{dd}, \mathrm{J}=7.8 ; 2.3 \mathrm{~Hz}, 1 \mathrm{H}, 4^{\prime}-\mathrm{H}\right), 6.91(\mathrm{~d}, \mathrm{~J}=2.1 \mathrm{~Hz}$, $\left.1 \mathrm{H}, 2^{\prime}-\mathrm{H}\right), 6.92\left(\mathrm{~d}, \mathrm{~J}=8.1 \mathrm{~Hz}, 1 \mathrm{H}, 6^{\prime}-\mathrm{H}\right), 7.12(\mathrm{~d}, \mathrm{~J}=12.1 \mathrm{~Hz}, 1 \mathrm{H}, \mathrm{CH}=), 7,17$ (t, J = 8.3 Hz, $1 \mathrm{H}$, 
5'-H), 7.18 (t, J = 9.9 Hz, $1 \mathrm{H}, 5-\mathrm{H}), 7.19(\mathrm{t}, \mathrm{J}=9.9 \mathrm{~Hz}, 1 \mathrm{H}, 7-\mathrm{H}), 7.29$ (d, J = 4.1 Hz, $1 \mathrm{H}, 3-\mathrm{H}), 7.63$ $(\mathrm{t}, \mathrm{J}=9.8 \mathrm{~Hz}, 1 \mathrm{H}, 6-\mathrm{H}), 7.80(\mathrm{~d}, \mathrm{~J}=4.1 \mathrm{~Hz}, 1 \mathrm{H}, 2-\mathrm{H}), 8.30(\mathrm{~d}, \mathrm{~J}=9.3 \mathrm{~Hz}, 1 \mathrm{H}, 4-\mathrm{H}), 8.43(\mathrm{~d}, \mathrm{~J}=9.8$ $\mathrm{Hz}, 1 \mathrm{H}, 8-\mathrm{H}) .{ }^{13} \mathrm{C}-\mathrm{NMR}(75 \mathrm{MHz}): \delta(\mathrm{ppm})=55.23$ (Me), 113.7 (C6'), 114.3 (C4'), 118.7 (C3), 121.8 (C2'), 123.8 (C7), 123.9 (C5), 124.8 (CHAz), 126.6 (C1), 128.8 (CHPh), 130.1 (C5'), 134.9 (C8), 137.4 (C1'), 137.5 (C4), 137.6 (C2), 139.0 (C6), 140.8 (C8a), 142.9 (C3a), 160.6 (C3'). IR: 433, 466, 504, 546, 585, 686, 725, 783, 819, 863, 882, 909, 947, 957, 1033, 1078, 1147, 1183, 1236, 1251, 1311, $1392,1435,1459,1483,1525,1562,1581,1615,2837,2932,2965,3009 \mathrm{~cm}^{-1}$. MS [GC] (m/z): 261 [M+1]. Calcd. for $\mathrm{C}_{19} \mathrm{H}_{16} \mathrm{O}: \mathrm{C}, 87.66 ; \mathrm{H}, 6.19 ; \mathrm{O}, 6.15$. Found: $\mathrm{C}, 87.68 ; \mathrm{H}, 6.17$.

1-[(E)-2-(3-Methylphenyl)ethenyl]azulene, $E-2 b$. Green crystals, m.p. $84^{\circ} \mathrm{C}$. UV-Viz, $\lambda_{\max }(\lg \varepsilon)$ : 255 (4.43), 305 (4.44), 316 (4.53), 334 (4.41), 378 (4.31), 396 (4.37), 640 (2.57) nm. ${ }^{1} \mathrm{H}-\mathrm{NMR}(500$ $\mathrm{MHz}): \delta(\mathrm{ppm})=2.36(\mathrm{~s}, 3 \mathrm{H}, \mathrm{Me}), 7.05\left(\mathrm{~d}, \mathrm{~J}=7.4 \mathrm{~Hz}, 1 \mathrm{H}, 4^{\prime}-\mathrm{H}\right), 7.14(\mathrm{t}, \mathrm{J}=9.6 \mathrm{~Hz}, 1 \mathrm{H}, 5-\mathrm{H}), 7.18$ $(\mathrm{t}, \mathrm{J}=9.6 \mathrm{~Hz}, 1 \mathrm{H}, 7-\mathrm{H}), 7,25\left(\mathrm{dd}, \mathrm{J}=7.7 \mathrm{~Hz}, \mathrm{~J}=7.4 \mathrm{~Hz}, 1 \mathrm{H}, 5^{\prime}-\mathrm{H}\right), 7.26(\mathrm{~d}, \mathrm{~J}=16.9 \mathrm{~Hz}, 1 \mathrm{H}, \mathrm{CH})$, 7.45 (d, J = 4.1 Hz, 1 H, 3-H), 7.47 (d, J = 7.7 Hz, 1 H, 6'-H), 7.52 (s, 1 H, 2'-H), 7.60 (t, J = 9.6 Hz, 1 H, 6-H), 7.90 (d, J = 16.1 Hz, $1 \mathrm{H}, \mathrm{CH}), 8.28$ (d, J = 9.2 Hz, $1 \mathrm{H}, 4-\mathrm{H}), 8.34$ (d, J = 4.2 Hz, $1 \mathrm{H}, 2-\mathrm{H})$, $8.69(\mathrm{~d}, \mathrm{~J}=9.8 \mathrm{~Hz}, 1 \mathrm{H}, 8-\mathrm{H}) .{ }^{13} \mathrm{C}-\mathrm{NMR}(75 \mathrm{MHz}): \delta(\mathrm{ppm})=21.48(\mathrm{Me}), 120.0(\mathrm{C} 3), 121.4(\mathrm{CHAz})$, 123.6 (C7), 124.2 (C6’), 124.7 (C5), 127.4 (CHPh), 127.5 (C2'), 128.4 (C6'), 128.5 (C1), 129.4 (C5'), 134.0 (C2), 134.8 (C8), 136.4 (C3'), 137.6 (C4), 138.8 (C1'), 139.3 (C6), 139.4 (C8a), 144.0 (C3a). IR: 440, 581, 690, 728, 778, 791, 984, 1392, 1450, 1564, 1590, 1613, $28512919,3018 \mathrm{~cm}^{-1}$. MS [GC] (m/z): $245\left[\mathrm{M}^{+}+1\right]$. Calcd. for $\mathrm{C}_{19} \mathrm{H}_{16}: \mathrm{C}, 93.40 ; \mathrm{H}, 6.60$. Found: $\mathrm{C}, 93.42 ; \mathrm{H}, 6.58$.

1-[(Z)-2-(3-Methylphenyl)ethenyl]azulene, Z-2b. Green crystals, m.p. $45^{\circ} \mathrm{C}$. UV-Viz, $\lambda_{\max }(\lg \varepsilon)$ : 218 (4.18), 258 (4.44), 309 (4.55), 388 (3.82), 618 (2.39) nm. ${ }^{1} \mathrm{H}-\mathrm{NMR}(500 \mathrm{MHz}): \delta$ (in ppm) $=2.23$ (s, $3 \mathrm{H}, \mathrm{Me}), 6.64$ (d, J = 12.2 Hz, $1 \mathrm{H}, \mathrm{CH}=), 7.03$ (d, J = 6.4 Hz, $\left.1 \mathrm{H}, 4^{\prime}-\mathrm{H}\right), 7.10$ (d, J = 12.2 Hz, 1 $\mathrm{H}, \mathrm{CH}=), 7.17(\mathrm{t}, \mathrm{J}=9.6 \mathrm{~Hz}, 1 \mathrm{H}, 5-\mathrm{H}), 7.18(\mathrm{t}, \mathrm{J}=9.6 \mathrm{~Hz}, 1 \mathrm{H}, 7-\mathrm{H}), 7.26(\mathrm{~d}, \mathrm{~J}=4.0 \mathrm{~Hz}, 1 \mathrm{H}, 3-\mathrm{H})$, $7,30\left(\mathrm{dd}, \mathrm{J}=7.7 \mathrm{~Hz}, \mathrm{~J}=7.4 \mathrm{~Hz}, 1 \mathrm{H}, 5^{\prime}-\mathrm{H}\right), 7.38\left(\mathrm{~d}, \mathrm{~J}=1.1 \mathrm{~Hz}, 1 \mathrm{H}, 2^{\prime}-\mathrm{H}\right), 7.38$ (dd, J = 6.4; $1.3 \mathrm{~Hz}, 1$ H, 6'-H), $7.62(\mathrm{t}, \mathrm{J}=9.8 \mathrm{~Hz}, 1 \mathrm{H}, 6-\mathrm{H}), 7.76(\mathrm{~d}, \mathrm{~J}=4.2 \mathrm{~Hz}, 1 \mathrm{H}, 2-\mathrm{H}), 8.28(\mathrm{~d}, \mathrm{~J}=9.3 \mathrm{~Hz}, 1 \mathrm{H}, 4-\mathrm{H})$, $8.43(\mathrm{~d}, \mathrm{~J}=9.8 \mathrm{~Hz}, 1 \mathrm{H}, 8-\mathrm{H}) .{ }^{13} \mathrm{C}-\mathrm{NMR}(75 \mathrm{MHz}): \delta($ in ppm) $=21.35(\mathrm{Me}), 118.7(\mathrm{C} 3), 123.5(\mathrm{C} 7)$, 123.7 (C6'), 124.8 (C5), 126.3 (CHAz), 128.5 (CHPh), 126.7 (C1), 129.0 (C2'), 129.5 (C4'), 129.7 (C6'), 130.0 (C5'), 134.3 (C2), 134.5 (C8), 134.8 (C3'), 137.6 (C4), 137.4 (C1'), 139.0 (C6), 139.5 (C8a), 142.9 (C3a). IR: 492, 582, 690, 724, 742, 779, 795, 826, 850, 912, 943, 958, 996, 1023, 1049, $1067,1087,1297,1373,1392,1431,1457,1474,1526,1562,1593,2851,2920,3023,3042 \mathrm{~cm}^{-1}$. MS [GC] (m/z): $245\left[\mathrm{M}^{+}+1\right]$. Calcd. for $\mathrm{C}_{19} \mathrm{H}_{16}: \mathrm{C}, 93.40 ; \mathrm{H}, 6.60$. Found: C, 93.39; H, 6.61.

1-[(E)-2-(3-tTrifluoromethylphenyl)ethenyl]azulene, E-2c. Green crystals, m.p. $130^{\circ} \mathrm{C}$. UV-Viz, $\lambda_{\max }(\lg \varepsilon): 224 \operatorname{sh}(4.26), 255$ (4.45), 315 (4.48), 338sh(4.37), 397 (4.46), 412sh (4.30), 629 (2.54) nm. ${ }^{1} \mathrm{H}-\mathrm{NMR}(500 \mathrm{MHz}): \delta($ in ppm) $=7.20(\mathrm{t}, \mathrm{J}=9.6 \mathrm{~Hz}, 1 \mathrm{H}, 5-\mathrm{H}), 7.23(\mathrm{t}, \mathrm{J}=9.8 \mathrm{~Hz}, 1 \mathrm{H}, 7-\mathrm{H}), 7.40$ $(\mathrm{d}, \mathrm{J}=16.1 \mathrm{~Hz}, 1 \mathrm{H}, \mathrm{CH}=), 7.48(\mathrm{~d}, \mathrm{~J}=4.1 \mathrm{~Hz}, 1 \mathrm{H}, 3-\mathrm{H}), 7.54\left(\mathrm{~d}, \mathrm{~J}=7.7 \mathrm{~Hz}, 1 \mathrm{H}, 4^{\prime}-\mathrm{H}\right), 7.59(\mathrm{t}, \mathrm{J}=$ $\left.7.7 \mathrm{~Hz}, 1 \mathrm{H}, 5^{\prime}-\mathrm{H}\right), 7.67$ (t, J = 9.7 Hz, $\left.1 \mathrm{H}, 6-\mathrm{H}\right), 7.97$ (d, J = 7.7 Hz, $1 \mathrm{H}, 6$ '-H), 8.01 (bs, $\left.1 \mathrm{H}, 2^{\prime}-\mathrm{H}\right)$, $8.11(\mathrm{~d}, \mathrm{~J}=16.1 \mathrm{~Hz}, 1 \mathrm{H}, \mathrm{CH}=), 8.33(\mathrm{~d}, \mathrm{~J}=9.7 \mathrm{~Hz}, 1 \mathrm{H}, 4-\mathrm{H}), 8.38(\mathrm{~d}, \mathrm{~J}=4.2 \mathrm{~Hz}, 1 \mathrm{H}, 2-\mathrm{H}), 8.79(\mathrm{~d}$, $\mathrm{J}=9.9 \mathrm{~Hz}, 1 \mathrm{H}, 8-\mathrm{H}) .{ }^{13} \mathrm{C}-\mathrm{NMR}(75 \mathrm{MHz}): \delta=120.2(\mathrm{C} 3), 123.2\left(\mathrm{q}, \mathrm{J}^{\mathrm{CF}}=3.8 \mathrm{~Hz}, \mathrm{C} 2\right.$ '), $123.7\left(\mathrm{q}, \mathrm{J}^{\mathrm{CF}}\right.$ = 3.5 Hz, C4'), 123.7 (CH=), 124.1 (C7), 125.1 (C5), $125.4(\mathrm{C}=), 127.9$ (C1), 130.3 (C5', C6'), 130.3 (q, J = 31.6 Hz, CF3'), 134.2 (C2), 135.0 (C8), 137.0 (C1'), 137.9 (C4), 139.5 (C6), 140.7 (C8a), 144.2 (C3a). IR: 573, 654, 694, 738, 786, 802, 887, 948, 1069, 1091, 1106, 1129, 1160, 1199, 1358, 1397, 1435, 1489, 1566, 1606, 1696, 2032, 2340, 2361, 2851, $2921 \mathrm{~cm}^{-1}$. MS [GC] (m/z): 299 [M+1]. Calcd. for $\mathrm{C}_{19} \mathrm{H}_{15} \mathrm{~F}$ : C, 76.50; H, 4.39; F, 19.11. Found: C, 76.49; H, 4.41.

1-[(Z)-2-(3-Trifluoromethylphenyl)ethenyl]azulene, Z-2c. Green crystals, m.p. $106^{\circ} \mathrm{C}$. UV-Viz, $\lambda_{\max }(\lg \varepsilon): 256$ (4.27), 284 (4.31), 310 (4.39), 388 (4.07), 610 (2.41) nm. ${ }^{1} \mathrm{H}-\mathrm{NMR}$ (500 MHz): $\delta$ (in $\mathrm{ppm})=6.73(\mathrm{~d}, \mathrm{~J}=12.1 \mathrm{~Hz}, 1 \mathrm{H}, \mathrm{CH}=), 7.22(\mathrm{t}, \mathrm{J}=9.6 \mathrm{~Hz}, 1 \mathrm{H}, 5-\mathrm{H}), 7.24(\mathrm{t}, \mathrm{J}=9.8 \mathrm{~Hz}, 1 \mathrm{H}, 7-\mathrm{H})$, $7.26(\mathrm{~d}, \mathrm{~J}=12.1 \mathrm{~Hz}, 1 \mathrm{H}, \mathrm{CH}=), 7.30(\mathrm{~d}, \mathrm{~J}=4.1 \mathrm{~Hz}, 1 \mathrm{H}, 3-\mathrm{H}), 7.48(\mathrm{t}, \mathrm{J}=7.8 \mathrm{~Hz}, 1 \mathrm{H}, 5$ '- $\mathrm{H}), 7.54(\mathrm{~d}$, $\left.\mathrm{J}=7.8 \mathrm{~Hz}, 1 \mathrm{H}, 6^{\prime}-\mathrm{H}\right), 7.61\left(\mathrm{~d}, \mathrm{~J}=7.8 \mathrm{~Hz}, 1 \mathrm{H}, 4^{\prime}-\mathrm{H}\right), 7.66$ (bs, $\left.1 \mathrm{H}, 2^{\prime}-\mathrm{H}\right), 7.67$ (t, J = 9.9 Hz, $1 \mathrm{H}, 6-$ H), $7.69(\mathrm{~d}, \mathrm{~J}=4.2 \mathrm{~Hz}, 1 \mathrm{H}, 2-\mathrm{H}), 8.35(\mathrm{~d}, \mathrm{~J}=9.7 \mathrm{~Hz}, 1 \mathrm{H}, 4-\mathrm{H}), 8.45(\mathrm{~d}, \mathrm{~J}=9.9 \mathrm{~Hz}, 1 \mathrm{H}, 8-\mathrm{H}) .{ }^{13} \mathrm{C}-$ 
NMR (75 MHz): $\delta=118.9(\mathrm{C} 3), 124.1(\mathrm{CH}=), 124.2\left(\mathrm{q}, \mathrm{J}^{\mathrm{CF}}=3.8 \mathrm{~Hz}, \mathrm{C} 2\right.$ '), $125.2(\mathrm{C} 7), 125.6(\mathrm{C} 5)$, $125.8\left(\mathrm{q}, \mathrm{J}^{\mathrm{CF}}=4.0 \mathrm{~Hz}, \mathrm{C} 4\right.$ '), $126.9(\mathrm{C}=), 127.0(\mathrm{C} 1), 130.1\left(\mathrm{C} 5^{\prime}\right), 130.3$ (q, J = 31.6 Hz, CF3'), 133.0 (C6'), 135.1 (C2), 137.1 (C8), 137.7 (C1'), 137.8 (C4), 139.3 (C6), 140.6 (C8a), 143.0 (C3a). IR: 573, 651, 694, 736, 787, 905, 951, 1069, 1105, 1128, 1157, 1200, 1216, 1327, 1411,1433, 1461, 1485, 1530, 1565, 1607, 2034, 2360, 2581, 2920, $3062 \mathrm{~cm}^{-1}$. MS [GC] (m/z): $299\left[\mathrm{M}^{+}+1\right]$. Calcd. for $\mathrm{C}_{19} \mathrm{H}_{15} \mathrm{~F}$ : C, 76.50; H, 4.39; F, 19.11. Found: C, 76.47; H, 4.40.

1-[(E)-2-(3-Fluorophenyl)ethenyl]azulene, $E$-2d. Green crystals, m.p. $116^{\circ} \mathrm{C}$. UV-Viz, $\lambda \max (\lg \varepsilon)$ : 228 (4.29), 254 (4.49), 305 (4.45), 317 (4.55), 333 (4.45), 350 (4.41), 368 (4.37), 381 (4.41), 397 (4.48), 634 (2.54) nm. ${ }^{1} \mathrm{H}-\mathrm{NMR}(500 \mathrm{MHz}): \delta$ (in ppm) $=6.98\left(\mathrm{dt}, \mathrm{J}^{\mathrm{HF}}=8.4 ; \mathrm{J}=1.8 \mathrm{~Hz}, 1 \mathrm{H}, 2^{\prime}-\mathrm{H}\right)$, $7.18(\mathrm{t}, \mathrm{J}=9.4 \mathrm{~Hz}, 1 \mathrm{H}, 5-\mathrm{H}), 7.21(\mathrm{t}, \mathrm{J}=9.4 \mathrm{~Hz}, 1 \mathrm{H}, 7-\mathrm{H}), 7.30(\mathrm{~d}, \mathrm{~J}=16.1 \mathrm{~Hz}, 1 \mathrm{H}, \mathrm{CH}=), 7,38$ (dt, $\left.\mathrm{J}=8.0 \mathrm{~Hz}, \mathrm{~J}^{\mathrm{HF}}=6.2 \mathrm{~Hz}, 1 \mathrm{H}, 5^{\prime}-\mathrm{H}\right), 7.45(\mathrm{~d}, \mathrm{~J}=4.3 \mathrm{~Hz}, 1 \mathrm{H}, 3-\mathrm{H}), 7.47$ (d, J =7.2 Hz, 1 H, 4'-H), 7.51 (dt, J =10.9; $2.0 \mathrm{~Hz}, 1 \mathrm{H}, 6$ '-H), 7.64 (t, J = 9.8 Hz, $1 \mathrm{H}, 6-\mathrm{H}), 8.01$ (d, J = $16.1 \mathrm{~Hz}, 1 \mathrm{H}, \mathrm{CH}=), 8.32$ $(\mathrm{d}, \mathrm{J}=9.4 \mathrm{~Hz}, 1 \mathrm{H}, 4-\mathrm{H}), 8.35(\mathrm{~d}, \mathrm{~J}=4.3 \mathrm{~Hz}, 1 \mathrm{H}, 2-\mathrm{H}), 8.77$ (d, J = 9.8 Hz, $1 \mathrm{H}, 8-\mathrm{H}) .{ }^{13} \mathrm{C}-\mathrm{NMR}(75$ $\mathrm{MHz}): \delta($ in $\mathrm{ppm})=112.7\left(\mathrm{C}^{\prime}\right.$ ') $113.5\left(\mathrm{C}^{\prime}\right.$ '), $120.1(\mathrm{C} 3), 122.0(\mathrm{C}=), 123.2\left(\mathrm{C} 6{ }^{\prime}\right), 124.0(\mathrm{C} 5), 125.0$ (C7), 125.8 (C=), 128.0 (C1), 131.0 (C5'), 131.2 (C6'), 134.2 (C2), 134.9 (C8), 136.9 (C1'), 137.8 (C4), 139.4 (C6), 142.3 (C8a), 144.2 (C3a), 164.2 (C3'). IR: 455, 568, 586, 682, 740, 784, 878, 932, 951, 976, 1137, 1237, 1315, 1395, 1439, 1454, 1484, 1571, 1599, 1621, 2921, $3031 \mathrm{~cm}^{-1}$. MS [GC] (m/z): 249 [M+1]. Calcd. for $\mathrm{C}_{18} \mathrm{H}_{13} \mathrm{~F}: \mathrm{C}, 87.07 ; \mathrm{H}, 5.28 ; \mathrm{F}, 7.65$. Found: C, 87.08; H, 5.29.

1-[(Z)-2-(3-Fluorophenyl)ethenyl]azulene, Z-2d. Blue-green crystals, m.p. $50^{\circ} \mathrm{C}$. UV-Viz, $\lambda_{\max }(\lg$ $\varepsilon): 224$ (4.43), 256 (4.36), 282 (4.37), 310 (4.42), 388 (4.02), 612 (2.36) nm. ${ }^{1} \mathrm{H}-\mathrm{NMR}$ (500 MHz): $\delta$ (in ppm) $=6.65(\mathrm{~d}, \mathrm{~J}=12.1 \mathrm{~Hz}, 1 \mathrm{H}, \mathrm{CH}=), 6.97\left(\mathrm{dt}, \mathrm{J}^{\mathrm{HF}}=8.4 ; \mathrm{J}=1.8 \mathrm{~Hz}, 1 \mathrm{H}, 2^{\prime}-\mathrm{H}\right), 7.07(\mathrm{dt}, \mathrm{J}$ $\left.=10.5 ; 2.0 \mathrm{~Hz}, 1 \mathrm{H}, 4^{\prime}-\mathrm{H}\right), 7.18(\mathrm{t}, \mathrm{J}=9.4 \mathrm{~Hz}, 1 \mathrm{H}, 5-\mathrm{H}), 7.20(\mathrm{t}, \mathrm{J}=9.4 \mathrm{~Hz}, 1 \mathrm{H}, 7-\mathrm{H}), 7,24$ (dt, J = 8.0 $\left.\mathrm{Hz}, \mathrm{J}^{\mathrm{HF}}=6.2 \mathrm{~Hz}, 1 \mathrm{H}, 5^{\prime}-\mathrm{H}\right), 7.17(\mathrm{~d}, \mathrm{~J}=12.2 \mathrm{~Hz}, 1 \mathrm{H}, \mathrm{CH}=), 7.18(\mathrm{~d}, \mathrm{~J}=4.2 \mathrm{~Hz}, 1 \mathrm{H}, 3-\mathrm{H}), 7.38$ (dt, $\left.\mathrm{J}=10.9 ; 2.0 \mathrm{~Hz}, 1 \mathrm{H}, 6^{\prime}-\mathrm{H}\right), 7.63$ (t, J = 9.8 Hz, $\left.1 \mathrm{H}, 6-\mathrm{H}\right), 8.30$ (d, J = 9.4 Hz, $\left.1 \mathrm{H}, 4-\mathrm{H}\right), 7.76$ (d, J = $4.3 \mathrm{~Hz}, 1 \mathrm{H}, 2-\mathrm{H}), 8.42(\mathrm{~d}, \mathrm{~J}=9.8 \mathrm{~Hz}, 1 \mathrm{H}, 8-\mathrm{H}) .{ }^{13} \mathrm{C}-\mathrm{NMR}(75 \mathrm{MHz}): \delta=114.4$ (C2'), 115.6 (C4'), 118.9 (C3), 124.0 (C5), 125.0 (C7, C=), 125.4 (C6'), $127.4(\mathrm{C}=), 126.0$ (C1), 130.9 (C5'), 134.9 (C8), 137.3 (C2), 137.5 (C1'), 137.6 (C4), 139.1 (C6), 142.0 (C8a), 142.9 (C3a), 163.6(C3'). IR: 492, 511, 688,738, 781, 877, 922, 1089, 1123, 1181, 1228, 1249, 1394, 1431, 1458, 1476, 1500, 1567, 1602, 2581, 2921, 3024, $3062 \mathrm{~cm}^{-1}$. MS [GC] (m/z): $249\left[\mathrm{M}^{+}+1\right]$. Calcd. for $\mathrm{C}_{18} \mathrm{H}_{13} \mathrm{~F}: \mathrm{C}, 87.07 ; \mathrm{H}, 5.28 ; \mathrm{F}$, 7.65. Found: C, 87.09; H, 5.28.

1-[(E)-2-(3-Nitrophenyl)ethenyl]azulene, E-2e. Green crystals, m.p. $143^{\circ} \mathrm{C}$. UV-Viz, $\lambda_{\max }(\lg \varepsilon)$ : 215 (4.45), 285 (4.45), 306 (4.37), 388 (4.37) ,606 (2.57) nm. ${ }^{1} \mathrm{H}-\mathrm{NMR}(500 \mathrm{MHz}): \delta$ (in ppm) $=7.23$ $(\mathrm{t}, \mathrm{J}=9.5 \mathrm{~Hz}, 1 \mathrm{H}, 5-\mathrm{H}), 7.26(\mathrm{t}, \mathrm{J}=9.8 \mathrm{~Hz}, 1 \mathrm{H}, 7-\mathrm{H}), 7.64$ (t, J = 7.5 Hz, $1 \mathrm{H}, 5$ ' -H), 7.68 (d, J = 16.1 $\mathrm{Hz}, 1 \mathrm{H}, \mathrm{CH}=), 7.49$ (d, J = 4.2 Hz, $1 \mathrm{H}, 3-\mathrm{H}), 7.71$ (t, J = 9.8 Hz, $1 \mathrm{H}, 6-\mathrm{H}), 8.06$ (ddd, J = 8.1, 2.2, $0.9 \mathrm{~Hz}, 1 \mathrm{H}, 6$ '-H), 8.12 (d, J = 7.8, 0.7 Hz, $\left.1 \mathrm{H}, 4^{\prime}-\mathrm{H}\right), 8.18$ (d, J = 16.1 Hz, $\left.1 \mathrm{H}, \mathrm{CH}=\right), 8.36$ (d, J = $9.2 \mathrm{~Hz}, 1 \mathrm{H}, 4-\mathrm{H}), 8.40(\mathrm{~d}, \mathrm{~J}=4.2 \mathrm{~Hz}, 1 \mathrm{H}, 2-\mathrm{H}), 8.50\left(\mathrm{~d}, \mathrm{~J}=2.0 \mathrm{~Hz}, 1 \mathrm{H}, 2^{\prime}-\mathrm{H}\right), 8.82$ (d, J = 9.8 Hz, 1 $\mathrm{H}, 8-\mathrm{H}) .{ }^{13} \mathrm{C}-\mathrm{NMR}(75 \mathrm{MHz}): \delta=\left(\right.$ in ppm) 120.3 (C3), $121.0\left(\mathrm{C} 2^{\prime}\right), 121.6\left(\mathrm{C}^{\prime}\right.$ '), $124.4(\mathrm{C} 7), 124.5$ $(\mathrm{CH}=), 124.6(\mathrm{CH}=), 125.3(\mathrm{C} 5), 127.7(\mathrm{C} 1), 130.7\left(\mathrm{C}^{\prime}\right), 132.7\left(\mathrm{C}^{\prime}\right), 134.4(\mathrm{C} 2), 135.1(\mathrm{C} 8), 137.3$ (C8a), 138.0 (C4), 139.6 (C6), 141.6 (C1'), 144.4 (C3a), 149.9 (C3'). MS [GC] (m/z): 276 [M+1 ${ }^{+}+1$. Calcd. for $\mathrm{C}_{18} \mathrm{H}_{13} \mathrm{NO}_{2}$ : C, 78.53; H, 4.39; F, 19.11. Found: C, 76.54; H, 4.40.

1-[(Z)-2-(3-Nitrophenyl)ethenyl]azulene, Z-2e. Green crystals, m.p. $92^{\circ} \mathrm{C} .{ }^{1} \mathrm{H}-\mathrm{NMR}(500 \mathrm{MHz}): \delta$ = (in ppm) $6.76(\mathrm{~d}, \mathrm{~J}=12.1 \mathrm{~Hz}, 1 \mathrm{H}, \mathrm{CH}=), 7.25(\mathrm{t}, \mathrm{J}=9.8 \mathrm{~Hz}, 1 \mathrm{H}, 5-\mathrm{H}), 7.26(\mathrm{t}, \mathrm{J}=9.6 \mathrm{~Hz}, 1 \mathrm{H}, 7-$ H), $7.31(\mathrm{~d}, \mathrm{~J}=4.1 \mathrm{~Hz}, 1 \mathrm{H}, 3-\mathrm{H}), 7.32(\mathrm{~d}, \mathrm{~J}=16.1 \mathrm{~Hz}, 1 \mathrm{H}, \mathrm{CH}=), 7.54$ (t, J = 8.0 Hz, $1 \mathrm{H}, 5$ ' - H), 7.69 (t, J = 9.8 Hz, $1 \mathrm{H}, 6-\mathrm{H}), 7.73(\mathrm{~d}, \mathrm{~J}=4.2 \mathrm{~Hz}, 1 \mathrm{H}, 2-\mathrm{H}), 7.76\left(\mathrm{~d}, \mathrm{~J}=7.7,1 \mathrm{H}, 4^{\prime}-\mathrm{H}\right), 8.07$ (dd, J = 8.1, $\left.1.5 \mathrm{~Hz}, 1 \mathrm{H}, 6^{\prime}-\mathrm{H}\right), 8.20$ (d, J = $1.8 \mathrm{~Hz}, 1 \mathrm{H}, 2^{\prime}$-H), 8.36 (d, J = 9.4 Hz, $\left.1 \mathrm{H}, 4-\mathrm{H}\right), 8.48$ (d, J = 9.9 Hz, $1 \mathrm{H}, 8-\mathrm{H}) .{ }^{13} \mathrm{C}-\mathrm{NMR}$ (75 MHz): $\delta=$ (in ppm) 119.1 (C3), 122.4 (C6'), 123.7 (C2'), 124.3 (C5), 125.4 $(\mathrm{CH}=), 125.4(\mathrm{C} 1), 126.1(\mathrm{C} 7), 126.4(\mathrm{CH}=), 130.5(\mathrm{C} 5$ '), $135.1(\mathrm{C} 8), 135.6(\mathrm{CH}=), 137.1(\mathrm{C} 2), 137.9$ (C4), 139.4 (C6), 141.4 (C1'), 143.1 (C3a), 149.3 (C3'). MS [GC] (m/z): 276 [M+1]. Calcd. for $\mathrm{C}_{18} \mathrm{H}_{13} \mathrm{NO}_{2}$ : C, 78.53; H, 4.39; F, 19.11. Found: C, 76.52; H, 4.41. 
Benzyltriphenylarsonium iodide, 3a. White crystals, m.p. $156-7^{\circ} \mathrm{C}\left(155-7{ }^{\circ} \mathrm{C}\right.$, lit. [15]. ${ }^{1} \mathrm{H}-\mathrm{NMR}$ (300 MHz): $\delta=$ (in ppm) $5.23\left(\mathrm{CH}_{2}\right), 7.13\left(\mathrm{~d},{ }^{3} \mathrm{~J}=7.0 \mathrm{~Hz}, 2 \mathrm{H}, 2-\mathrm{H}, 6-\mathrm{H}\right), 7.24(\mathrm{t}, \mathrm{J}=7.0 \mathrm{~Hz}, 2 \mathrm{H}, 3-$ $\mathrm{H}, 5-\mathrm{H}), 7.27\left(\mathrm{t},{ }^{3} \mathrm{~J}=7.1 \mathrm{~Hz}, 1 \mathrm{H}, 4-\mathrm{H}\right), 7.62\left(\mathrm{~d},{ }^{3} \mathrm{~J}=7.7 \mathrm{~Hz}, 6 \mathrm{H}, 2^{\prime}-\mathrm{H}, 6{ }^{\prime}-\mathrm{H}\right), 7.72\left(\mathrm{t},{ }^{3} \mathrm{~J}=7.6 \mathrm{~Hz},{ }^{13} \mathrm{C}-\right.$ NMR $(75 \mathrm{MHz}): \delta=\left(\right.$ in ppm) $25.38\left(\mathrm{CH}_{2}\right), 120.8(\mathrm{C} 1$ '), $128.0(\mathrm{C} 4), 128.5(\mathrm{C} 2), 128.5(\mathrm{C} 6), 130.1$ (C3', C5'), 128.5 (C1), 132.6 (C2', C6'), 133.6 (C4'), 130.0 (C5), 130.0 (C3). MS [ESI] (m/z): 397 $\left[\mathrm{M}^{+}\right]$. Calcd. for $\mathrm{C}_{25} \mathrm{H}_{22} \mathrm{AsI}$ : C, 57.27; H, 4.23; As, 14.29; I, 24.21. Found: C, 57.30; H, 4.25.

3-Metoxybenzyltriphenylarsonium iodide, 3b. White crystals, m.p. $148^{\circ} \mathrm{C} .{ }^{1} \mathrm{H}-\mathrm{NMR}(500 \mathrm{MHz}): \delta$ $=\left(\right.$ in ppm) $3.72(\mathrm{~s}, \mathrm{OMe}), 5.18\left(\mathrm{~s}, \mathrm{CH}_{2}\right), 6.63(\mathrm{bs}, 1 \mathrm{H}, 2-\mathrm{H}), 6.74\left(\mathrm{~d},{ }^{3} \mathrm{~J}=7.6 \mathrm{~Hz}, 1 \mathrm{H}, 6-\mathrm{H}\right), 6.85(\mathrm{dd}$, $\left.{ }^{3} \mathrm{~J}=8.2 \mathrm{~Hz},{ }^{4} \mathrm{~J}=2.0 \mathrm{~Hz}, 1 \mathrm{H}, 4-\mathrm{H}\right), 7.17\left(\mathrm{t},{ }^{3} \mathrm{~J}=7.9 \mathrm{~Hz}, 1 \mathrm{H}, 5-\mathrm{H}\right), 7.63\left(\mathrm{~d},{ }^{3} \mathrm{~J}=7.6 \mathrm{~Hz}, 6 \mathrm{H}, 2^{\prime}-\mathrm{H}, 6{ }^{\prime}-\right.$ $\mathrm{H}), 7.73\left(\mathrm{t},{ }^{3} \mathrm{~J}=7.7 \mathrm{~Hz}, 6 \mathrm{H}, 3^{\prime}\right.$-H, 5'-H), 7.84 (t, $\left.{ }^{3} \mathrm{~J}=7.3 \mathrm{~Hz}, 3 \mathrm{H}, 4{ }^{\prime}-\mathrm{H}\right) .{ }^{13} \mathrm{C}-\mathrm{NMR}(75 \mathrm{MHz}): \delta$ (in $\mathrm{ppm})=30.85\left(\mathrm{CH}_{2}\right), 54.91(\mathrm{OMe}) 114.5(\mathrm{C} 4), 115.6(\mathrm{C} 2), 121.2\left(\mathrm{C}^{\prime}{ }^{\prime}\right), 122.5(\mathrm{C} 6), 130.6\left(\mathrm{C}^{\prime}, \mathrm{C}^{\prime}\right)$, 130.0 (C5), 130.2 (C1), 133.1 (C2', C6'), 134.0 (C4'), 159.2 (C3). MS [ESI] (m/z): 427 [M+']. Calcd. for $\mathrm{C}_{26} \mathrm{H}_{24}$ AsIO: C, 56.34; H, 4.36; As, 13.52; I, 22.89; O, 2.89. Found: C, 56.34; H, 4.38.

3-Methylbenzyltriphenylarsonium iodide, 3c. White crystals, m.p. $147^{\circ} \mathrm{C} .{ }^{1} \mathrm{H}-\mathrm{NMR}(500 \mathrm{MHz}): \delta=$ (in ppm) $2.08(\mathrm{Me}), 5.15\left(\mathrm{CH}_{2}\right), 6.80(\mathrm{bs}, 1 \mathrm{H}, 2-\mathrm{H}), 6.94\left(\mathrm{~d},{ }^{3} \mathrm{~J}=7.6 \mathrm{~Hz}, 1 \mathrm{H}, 6-\mathrm{H}\right), 7.10\left(\mathrm{~d},{ }^{3} \mathrm{~J}=8.2\right.$ $\mathrm{Hz}, 1 \mathrm{H}, 4-\mathrm{H}), 7.16$ (t, J = 7.9 Hz, $1 \mathrm{H}, 5-\mathrm{H}), 7.61$ (d, ${ }^{3} \mathrm{~J}=7.6 \mathrm{~Hz}, 6 \mathrm{H}, 2^{\prime}-\mathrm{H}, 6$ ' $\left.-\mathrm{H}\right), 7.73$ (t, ${ }^{3} \mathrm{~J}=7.7 \mathrm{~Hz}$, $6 \mathrm{H}, 3^{\prime}-\mathrm{H}, 5$ '-H), $7.84\left(\mathrm{t},{ }^{3} \mathrm{~J}=7.3 \mathrm{~Hz}, 3 \mathrm{H}, 4{ }^{\prime}-\mathrm{H}\right) .{ }^{13} \mathrm{C}-\mathrm{NMR}(75 \mathrm{MHz}): \delta($ in ppm $)=20.76(\mathrm{Me}), 30.82$ $\left(\mathrm{CH}_{2}\right), 121.2$ (C1'), 127.4 (C4), 131.2 (C2), 128.7 (C6), 130.5 (C3', C5'), 129.0 (C5), 128.7 (C1),

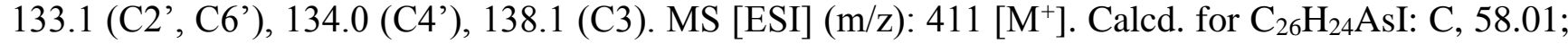
H, 4.49; As, 13.92; I, 23.58. Found: C, 57.98; H, 4.52.

3-Trifluoromethylbenzyltriphenylarsonium iodide, 3d. White crystals, m.p. $164^{\circ} \mathrm{C} .{ }^{1} \mathrm{H}-\mathrm{NMR}(500$ $\mathrm{MHz}): \delta=\left(\right.$ in ppm) $5.34\left(\mathrm{~s}, \mathrm{CH}_{2}\right), 7.35(\mathrm{bs}, 1 \mathrm{H}, 2-\mathrm{H}), 7.52\left(\mathrm{bd},{ }^{3} \mathrm{~J}=7.6 \mathrm{~Hz}, 2 \mathrm{H}, 4-\mathrm{H}, 6-\mathrm{H}\right), 7.63-7.66$ (m, $1 \mathrm{H}, 5-\mathrm{H}), 7.65\left(\mathrm{~d},{ }^{3} \mathrm{~J}=7.6 \mathrm{~Hz}, 6 \mathrm{H}, 2^{\prime}-\mathrm{H}, 66^{\prime}-\mathrm{H}\right), 7.73\left(\mathrm{t},{ }^{3} \mathrm{~J}=7.7 \mathrm{~Hz}, 6 \mathrm{H}, 3^{\prime}-\mathrm{H}, 5^{\prime}-\mathrm{H}\right), 7.85\left(\mathrm{t},{ }^{3} \mathrm{~J}=\right.$ $\left.7.3 \mathrm{~Hz}, 3 \mathrm{H}, 4^{\prime}-\mathrm{H}\right) .{ }^{13} \mathrm{C}-\mathrm{NMR}(75 \mathrm{MHz}): \delta=\left(\right.$ in ppm) $30.63\left(\mathrm{CH}_{2}\right), 121.2\left(\mathrm{C} 1^{\prime}\right), 134.7(\mathrm{C} 5), 124.1(\mathrm{~J}=$ $\left.272.7 \mathrm{CF}_{3}\right), 130.6(\mathrm{C} 6), 125.5(\mathrm{~J}=3.7 \mathrm{~Hz} \mathrm{C} 2), 127.7(\mathrm{~J}=3.7 \mathrm{~Hz}, \mathrm{C} 4), 129.8(\mathrm{~J}=32.0 \mathrm{~Hz}, \mathrm{C} 3), 131.1$ (C3', C5'), 131.9 (C1), 133.5 (C2', C6'), 134.6 (C4'). MS [ESI] (m/z): 465 [M+']. Calcd. for $\mathrm{C}_{26} \mathrm{H}_{21} \mathrm{AsF}_{3} \mathrm{I}: \mathrm{C}, 52.73 ; \mathrm{H}, 3.57$; As, 12.65; F, 9.62; I, 23.58. Found: C, 52.71; H, 3.59.

3-Fluorobenzyltriphenylarsonium iodide, 3e. White crystals, m.p. $168^{\circ} \mathrm{C} .{ }^{1} \mathrm{H}-\mathrm{NMR}(500 \mathrm{MHz}): \delta=$ (in ppm) $5.30\left(\mathrm{~s}, \mathrm{CH}_{2}\right), 6.93\left(\mathrm{dt},{ }^{\mathrm{HF}} \mathrm{J}=9.8 \mathrm{~Hz},{ }^{4} \mathrm{~J}=1 \mathrm{~Hz}, 1 \mathrm{H}, 2-\mathrm{H}\right), 7.00\left(\mathrm{~d},{ }^{3} \mathrm{~J}=7.7 \mathrm{~Hz}, 1 \mathrm{H}, 6-\mathrm{H}\right)$, $7.13\left(\mathrm{dt},{ }^{\mathrm{HF}} \mathrm{J}=8.5 \mathrm{~Hz},{ }^{4} \mathrm{~J}=2.2 \mathrm{~Hz}, 1 \mathrm{H}, 4-\mathrm{H}\right), 7.30\left(\mathrm{dt},{ }^{\mathrm{HF}} \mathrm{J}=6.2 \mathrm{~Hz},{ }^{3} \mathrm{~J}=7.9 \mathrm{~Hz}, 1 \mathrm{H}, 5-\mathrm{H}\right), 7.66\left(\mathrm{~d},{ }^{3} \mathrm{~J}\right.$ $\left.=7.6 \mathrm{~Hz}, 6 \mathrm{H}, 2^{\prime}-\mathrm{H}, 6{ }^{\prime}-\mathrm{H}\right), 7.73\left(\mathrm{t},{ }^{3} \mathrm{~J}=7.7 \mathrm{~Hz}, 6 \mathrm{H}, 3^{\prime}-\mathrm{H}, 5^{\prime}-\mathrm{H}\right), 7.84$ (t, $\left.{ }^{3} \mathrm{~J}=7.2 \mathrm{~Hz}, 3 \mathrm{H}, 4^{\prime}-\mathrm{H}\right) .{ }^{13} \mathrm{C}-$ NMR (75 MHz): $\delta=$ (in ppm) $30.22\left(\mathrm{~s}, \mathrm{CH}_{2}\right), 115.4\left(\mathrm{~d}, \mathrm{~J}^{\mathrm{CF}}=20.8 \mathrm{~Hz}, \mathrm{C} 4\right), 117.2\left(\mathrm{~d}, \mathrm{~J}^{\mathrm{CF}}=22.5 \mathrm{~Hz}\right.$, C2), 120.9 (s, C1'), 126.5 (d, J $\left.{ }^{\mathrm{CF}}=2.4 \mathrm{~Hz}, \mathrm{C} 6\right), 130.6\left(\mathrm{~s}, \mathrm{C}^{\prime}\right.$ ', C5'), $131.0\left(\mathrm{~d}, \mathrm{~J}^{\mathrm{CF}}=8.6 \mathrm{~Hz}, \mathrm{C} 5\right), 131.7$ $\left(\mathrm{d}, \mathrm{J}^{\mathrm{CF}}=8.2 \mathrm{~Hz}, \mathrm{C} 1\right), 133.1\left(\mathrm{~s}, \mathrm{C} 2^{\prime}, \mathrm{C}^{\prime}\right), 134.1\left(\mathrm{~s}, \mathrm{C} 4^{\prime}\right), 161.8\left(\mathrm{~d}, \mathrm{~J}^{\mathrm{CF}}=245.0 \mathrm{~Hz}, \mathrm{C} 3\right) . \mathrm{MS}$ [ESI] $(\mathrm{m} / \mathrm{z})$ : 415 [M+ $\mathrm{M}^{+}$. Calcd. for $\mathrm{C}_{25} \mathrm{H}_{21}$ AsFI: C, 55.37; H, 3.90; As, 13.82; I, 23.40; F, 3.50. Found: C, 55.35; H, 3.93 .

3-Nitrobenzyltriphenylarsonium iodide, 3f. White crystals, m.p. $166^{\circ} \mathrm{C} .{ }^{1} \mathrm{H}-\mathrm{NMR}(500 \mathrm{MHz}): \delta=$ (in ppm) $5.34\left(\mathrm{~s}, \mathrm{CH}_{2}\right), 7.57(\mathrm{t}, \mathrm{J}=7.7 \mathrm{~Hz}, 1 \mathrm{H}, 5-\mathrm{H}), 7.59\left(\mathrm{~d},{ }^{3} \mathrm{~J}=7.6 \mathrm{~Hz}, 1 \mathrm{H}, 6-\mathrm{H}\right), 7.65\left(\mathrm{~d},{ }^{3} \mathrm{~J}=7.6\right.$ $\left.\mathrm{Hz}, 6 \mathrm{H}, 2^{\prime}-\mathrm{H}, 6^{\prime}-\mathrm{H}\right), 7.73$ (t, $\left.{ }^{3} \mathrm{~J}=7.6 \mathrm{~Hz}, 6 \mathrm{H}, 3^{\prime}-\mathrm{H}, 5^{\prime}-\mathrm{H}\right), 7.85$ (t, $\left.{ }^{3} \mathrm{~J}=7.3 \mathrm{~Hz}, 3 \mathrm{H}, 6^{\prime}-\mathrm{H}\right), 7.91$ (bs, 1 $\mathrm{H}, 2-\mathrm{H}), 8.15\left(\mathrm{~d},{ }^{3} \mathrm{~J}=7.5 \mathrm{~Hz}, 1 \mathrm{H}, 4-\mathrm{H}\right) .{ }^{13} \mathrm{C}-\mathrm{NMR}(75 \mathrm{MHz}): \delta=\left(\right.$ in ppm) $29.88\left(\mathrm{CH}_{2}\right), 120.6(\mathrm{C} 1$ '), 123.3 (C4), 125.2 (C2), 130.6 (C6), 130.7 (C3', C5'), 131.4 (C1), 133.1 (C2', C6'), 134.2 (C4'), 136.7 (C5), 147.6 (C3). MS [ESI] (m/z): 442 [M+ $\mathrm{M}^{+}$. Calcd. for $\mathrm{C}_{25} \mathrm{H}_{21} \mathrm{AsINO}_{2}$ : C, 57.75; H, 3.72; As, 13.16; I, 22.29; N, 2.46; O, 5.62. Found: C, 57.73; H, 3.73.

4-Nitrobenzyltriphenylarsonium bromide, 4. White crystals, m.p, $150-151^{\circ} \mathrm{C}$, (lit. [16] $151{ }^{\circ} \mathrm{C}$, lit. [17] $\left.159{ }^{\circ} \mathrm{C}\right) .{ }^{1} \mathrm{H}-\mathrm{NMR}(300 \mathrm{MHz}): \delta=\left(\right.$ in ppm) $5.99\left(\mathrm{CH}_{2}\right), 7.56\left(\mathrm{t},{ }^{3} \mathrm{~J}=7.6 \mathrm{~Hz}, 6 \mathrm{H}, 3^{\prime}-\mathrm{H}, 5^{\prime}-\mathrm{H}\right)$, $7.58\left(\mathrm{~d},{ }^{3} \mathrm{~J}=7.0 \mathrm{~Hz}, 2 \mathrm{H}, 2-\mathrm{H}, 6-\mathrm{H}\right), 7.68\left(\mathrm{t},{ }^{3} \mathrm{~J}=7.5 \mathrm{~Hz}, 3 \mathrm{H}, 6{ }^{\prime}-\mathrm{H}\right), 7.73$ (d, ${ }^{3} \mathrm{~J}=7.7 \mathrm{~Hz}, 6 \mathrm{H}, 2^{\prime}-\mathrm{H}, 6$ ' $\mathrm{H}), 7.83(\mathrm{t}, \mathrm{J}=8.5 \mathrm{~Hz}, 2 \mathrm{H}, 3-\mathrm{H}, 5-\mathrm{H}) .{ }^{13} \mathrm{C}-\mathrm{NMR}(75 \mathrm{MHz}): \delta=\left(\right.$ in ppm) $32.24\left(\mathrm{CH}_{2}\right), 120.4\left(\mathrm{C} 1^{\prime}\right)$, 123.5 (C2,C6), 130.8 (C3',C5'), 132.4 (C3,C5), 133.5 (C2',C6'), 134.2 (C4'), 136.8 (C1), 147.5 (C4). 
MS [ESI] (m/z): $442\left[\mathrm{M}^{+}\right]$. Calcd. for $\mathrm{C}_{25} \mathrm{H}_{21} \mathrm{AsINO}_{2}$ : C, 57.75; H, 3.72; As, 13.16; I, 22.29; N, 2.46; O, 5.62. Found: C, 57.71; H, 3.74.

1-[(E)-2-(4-Nitrophenyl)ethenyl $]$ azulene, 5a. Brown crystals, m.p. $191-192^{\circ} \mathrm{C}\left(187{ }^{\circ} \mathrm{C}\right.$ lit. $\left.[2]\right) .{ }^{1} \mathrm{H}-$ $\operatorname{NMR}(300 \mathrm{MHz}): \delta=($ in ppm) $7.20(\mathrm{~d}, \mathrm{~J}=16.3 \mathrm{~Hz}, 1 \mathrm{H}, \mathrm{CH}=), 7.20(\mathrm{t}, \mathrm{J}=9.5 \mathrm{~Hz}, 1 \mathrm{H}, 5-\mathrm{H}), 7.23(\mathrm{t}$, $\mathrm{J}=9.8 \mathrm{~Hz}, 1 \mathrm{H}, 7-\mathrm{H}), 7.45(\mathrm{~d}, \mathrm{~J}=4.3 \mathrm{~Hz}, 1 \mathrm{H}, 3-\mathrm{H}), 7.62(\mathrm{t}, \mathrm{J}=9.9 \mathrm{~Hz}, 1 \mathrm{H}, 6-\mathrm{H}), 7.67(\mathrm{~d}, \mathrm{~J}=8.8 \mathrm{~Hz}$, 2 H, 3'-H, 5'-H), 7.89 (d, J = 16 Hz, 1 H, CH=), 8.23 (d, J = 8.8 Hz, 2 H, 2'-H, 6'-H), 8.27 (d, J = 9.2 $\mathrm{Hz}, 1 \mathrm{H}, 4-\mathrm{H}), 8.28(\mathrm{~d}, \mathrm{~J}=4.3 \mathrm{~Hz}, 1 \mathrm{H}, 2-\mathrm{H}), 8.54(\mathrm{~d}, \mathrm{~J}=9.8 \mathrm{~Hz}, 1 \mathrm{H}, 8-\mathrm{H}) .{ }^{13} \mathrm{C}-\mathrm{NMR}(75 \mathrm{MHz}): \delta=$ (in ppm) 119.8 (C3), $123.7(\mathrm{AzC}=), 124.0(\mathrm{C} 2), 124.4$ (C2', C6'), 125.0 (C5), 125.2 (C7), 126.1 (C3', C5'), 126.4 (C1), 133.7 ( $\mathrm{PhC}=, \mathrm{C} 4), 136,9$ (C3a), 137.4 (C6), 138.8 (C8), 143.2 (C8a), 145.2 (C4'), $145.9\left(\mathrm{C} 1^{\prime}\right)$. MS [ESI] (m/z): $276\left[\mathrm{M}^{+}+1\right]$. Calcd. for: $\mathrm{C}_{18} \mathrm{H}_{13} \mathrm{NO}_{2}: \mathrm{C}, 78.53 ; \mathrm{H}, 4.76 ; \mathrm{N}, 5.09 ; \mathrm{O}$, 11.62. Found: C, 78.50; H, 4.77 .

1-[(E)-2-(4-Nitrophenyl)ethenyl]-4,6,8-trimethylazulene, 5b. Brown crystals, m.p. $165^{\circ} \mathrm{C},{ }^{1} \mathrm{H}-\mathrm{NMR}$ (300 MHz): $\delta=($ in ppm) 2.59 (s, 3H, Me $), 2.85$ (s, $\left.3 \mathrm{H}, \mathrm{Me}_{4}\right), 3.10$ (s, $\left.3 \mathrm{H}, \mathrm{Me}_{8}\right), 6.95(\mathrm{~d}, \mathrm{~J}=15.8 \mathrm{~Hz}$, $1 \mathrm{H}, \mathrm{PhCH}=), 7.04$ (s, $2 \mathrm{H}, 5-\mathrm{H}, 7-\mathrm{H}), 7.36$ (d, J = 4.3 Hz, 3-H), 7.57 (d, J = 8.8 Hz, 2 H, 3'-H, 5'-H), $7.95(\mathrm{~d}, \mathrm{~J}=4.2 \mathrm{~Hz}, 1 \mathrm{H}, 2-\mathrm{H}), 8.19$ (d, J = 8.8 Hz, $\left.2 \mathrm{H}, 2^{\prime}-\mathrm{H}, 6^{\prime}-\mathrm{H}\right), 8.20(\mathrm{~d}, \mathrm{~J}=15.8 \mathrm{~Hz}, 1 \mathrm{H}$, $\mathrm{AzCH}=) .{ }^{13} \mathrm{C}-\mathrm{NMR}(75 \mathrm{MHz}): \delta=\left(\right.$ in ppm) $25.80\left(\mathrm{Me}_{4}\right), 28.59\left(\mathrm{Me}_{8}\right), 29.74\left(\mathrm{Me}_{6}\right), 117.3(\mathrm{C} 3), 124.0$ $(\mathrm{AzCH}=), 124.3$ (C2', C6'), 126.0 (C3', C5'), 128.3 (C1), 128.9 (C2), 130.6 (C5), 131.8 (C7), 133.0 $(\mathrm{PhCH}=), 133.5$ (C3a), 139.8 (C8a), 145.6 (C4), 145.8 (C4'), 146.3 (C6), 147.3 (C8), 147.4 (C1'). MS [ESI] $(\mathrm{m} / \mathrm{z}): 318\left[\mathrm{M}^{+}+1\right]$. Calcd. for: $\mathrm{C}_{21} \mathrm{H}_{19} \mathrm{NO}_{2}: \mathrm{C}, 79.47 ; \mathrm{H}, 6.03 ; \mathrm{N}, 4.41 ; \mathrm{O}, 10.08$. Found: $\mathrm{C}$, $79.46 ; \mathrm{H}, 6.04$.

1-[(E)-2-(4-Nitrophenyl)ethenyl]-3,8-dimethyl-5-isopropylazulene, 5c. Dark green crystals, m.p. $133^{\circ} \mathrm{C},{ }^{1} \mathrm{H}-\mathrm{NMR}(300 \mathrm{MHz}): \delta\left(\right.$ in ppm) $=1.28(\mathrm{~d}, \mathrm{~J}=6.9 \mathrm{~Hz}, 6 \mathrm{H}, \mathrm{CHMe}), 2.54\left(\mathrm{~s}, 3 \mathrm{H}, \mathrm{Me}_{3}\right), 2.98$ (sept, J = 6.8 Hz, 1 H, CHMe), 2.99 (s, $3 \mathrm{H}, \mathrm{Me}_{8}$ ), 6.87 (d, J = 15.4 Hz, $1 \mathrm{H}, \mathrm{PhCH=),} 6.89$ (d, J = 9.9 Hz, $1 \mathrm{H}, 7-\mathrm{H}), 7.20$ (d, J = 9.9 Hz, 1 H, 6-H), 7.47 (d, J = 8.8 Hz, 2 H, 3'-H, 5'-H), 7.86 (s, 1 H, 2-H), 7.97 (bs, $1 \mathrm{H}, 4-\mathrm{H}), 8.10$ (d, J = 8.8 Hz, $2 \mathrm{H}, 2$ '-H, 6'-H), 8.14 (d, J = 15.4 Hz, $1 \mathrm{H}, \mathrm{GuCH}=) .{ }^{13} \mathrm{C}-$ NMR (75 MHz): $\delta\left(\right.$ in ppm) = $13.20\left(\mathrm{Me}_{3}\right), 24.56(\mathrm{iPr}), 28.88\left(\mathrm{Me}_{8}\right), 37.91$ (iPr), $123.5(\mathrm{GuC}=), 124.4$ (C2', C6'), 125.3 (C1), 125.9 (C3', C5'), 127.1 (C3), 128.8 (C3a), 129.0 (C2), 130.5 (C7), 133.9 $(\mathrm{PhC}=), 134.3(\mathrm{C} 4), 135.5(\mathrm{C} 6), 141.6(\mathrm{C} 5), 142.8$ (C8), 145.6 (C8a), 145.8 (C4'), 146.1 (C1'). [ESI] $(\mathrm{m} / \mathrm{z}): 344\left[\mathrm{M}^{+}+1\right]$. Calcd. for: $\mathrm{C}_{23} \mathrm{H}_{23} \mathrm{NO}_{2}: \mathrm{C}, 79.97 ; \mathrm{H}, 6.71 ; \mathrm{N}, 4.05 ; \mathrm{O}, 9.26$. Found: $\mathrm{C}, 79.96 ; \mathrm{H}$, 6.72 .

\section{Results and discussions Syntheses}

The literature review reports only few styrylazulenes with phenyl having a substituent in meta position, for example, as a result of a Horner-Wadsworth-Emmons reaction on 1-guaiazulenecarbaldehyde [18]. The poor yields obtained and the incomplete products characterization suggested us to pay attention to the synthesis and properties of this class of 1-styrylazulenes.

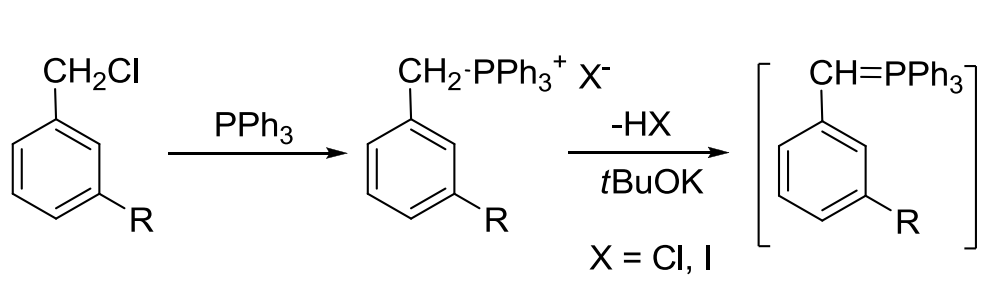

$1 R$

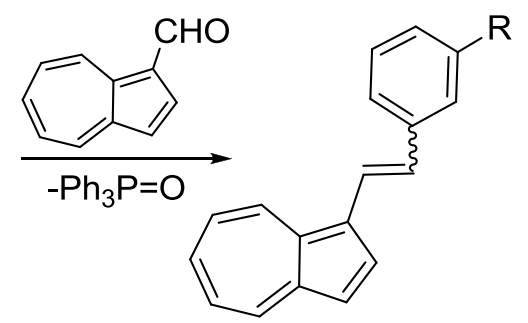

$\mathbf{2} \mathbf{R}(E$ and $Z)$

$\mathbf{R}=\mathbf{a}: \mathrm{OMe} ; \mathbf{b}: \mathrm{Me} ; \mathbf{c}: \mathrm{CF}_{3} ; \mathbf{d}: \mathrm{F} ; \mathbf{e}: \mathrm{NO}_{2}$

Scheme 1. Wittig synthesis of meta-substituted 1-styrylazulenes 
The synthesis of the desired compounds, shown in Scheme 1, followed the path described in the literature [1]. The meta-substituted benzyltriphenylphosphonium salts, 1R, were obtained in almost quantitative yields and characterized (compounds with $\mathbf{R}=\mathrm{OMe}$ and $\mathrm{NO}_{2}$ are commercially available). The ylides were prepared in the presence of strong base $(t \mathrm{BuOK})$ in $\mathrm{DCM}$ under nitrogen and without separation, a solution of 1-azulenecarbaldehyde in DCM was added. A mixture of $Z$ and $E$ alkenes, 2R, was generated in the good yields, as shown in Table 1, and an amount of unreacted 1-azulenecarbaldehydes was recovered in each experiment. The relatively small difference between the obtained yields, as well as between $Z / E$ isomers ratio seems to be the result of the low inductive effect exerted by substituents in the meta position towards reaction center.

Table 1. Wittig reaction yields starting from benzyltriphenylphosphonium salts, 1R (in \%)

\begin{tabular}{|c|c|c|c|c|}
\hline \multirow{2}{*}{$\begin{array}{l}\text { Starting } \\
\text { Compds. }\end{array}$} & \multirow[t]{2}{*}{$\mathrm{R}$} & \multicolumn{2}{|c|}{ Isomers of alkene $\mathbf{2 R}$} & \multirow{2}{*}{$\begin{array}{l}\text { Unreacted } \\
\text { aldehyde }\end{array}$} \\
\hline & & $Z$ & $E$ & \\
\hline $1 a^{*}$ & $\mathrm{OMe}$ & 35 & 22 & 12 \\
\hline $1 \mathrm{~b}$ & $\mathrm{Me}$ & 44 & 25 & 14 \\
\hline 1c & $\mathrm{CF}_{3}$ & 51 & 28 & 12 \\
\hline 1d & $\mathrm{F}$ & 54 & 30 & 10 \\
\hline $1 e^{*}$ & $\mathrm{NO}_{2}$ & 31 & 21 & 10 \\
\hline
\end{tabular}

"Salts commercially available.

In order to develop a research in the field of arsa-Wittig reaction, an effort was made in view of the synthesis of the arsonium salts. Reviews published about arsonium ylides, [6-8] in addition to the synthesis as such, also deal with the reaction mechanism and the use of the products for other reactions. A special importance was given to obtain alkenes after reaction with carbonylic derivatives, the arsa-Wittig condensation. However, this reaction is somewhat difficult because both alkenes and epoxides can be formed mainly depending on the starting compounds [19] and the reported results are not always consistent [20]. As represented in Scheme 2, regarding their stability, arsonium ylides can be divided into 3 categories.
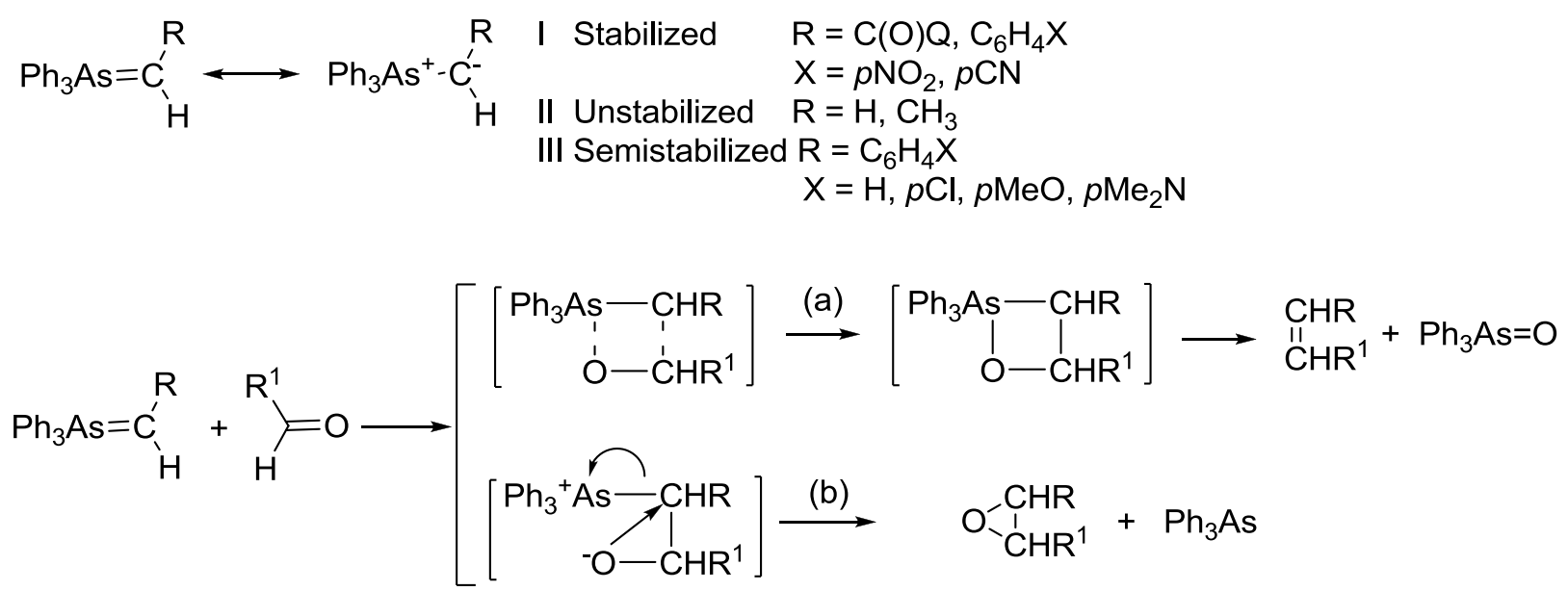

Scheme 2. Arsa-Wittig reaction

The two observed reaction pathways for the arsa-Wittig reaction were shown in Scheme 2. One pathway (route a) proceeds via an oxaarsetane, like the phospha-Wittig reaction, with olefins being formed as products. The other pathway (route b) is supposed to proceed via a betaine as intermediate, like the reaction of sulfur-ylides with carbonyl functions leading to epoxides [21].

For stabilized and unstabilized ylides the behavior is clear, only one product was obtained: alkene or epoxide, respectively. The condensation of semi-stabilized ylides, compounds III, occurs more complicated and the literature data are not coherent in this regard. Thus, Kendurkar in the preamble of a paper [19] stated that 4-nitrobenzyltriphenylarsonium ylide is semi-stabilized giving after arsa-Wittig 
condensation with aldehydes a mixture of alkene and epoxide. However, from the experimental work resulted that only alkene was formed, in yield over $90 \%$, after this condensation. Depending on structure of staring compounds, and reaction conditions, mainly the used base and solvent, the resulted mixture can contain olefins [22], epoxides [23] or both compounds [24,25]. It should be noted that all products, epoxides or alkenes were always generated as trans isomers and exceptionally is also formed small amount of cis isomers [24,25]. A reduced influence was found with respect to phenyl replacement in triphenylarsonium ylides [25]. At the same time, the solvents used can influence the product ratio: while polar protic solvents, such as $\mathrm{EtOH}$, favor the epoxide formation, due to the ionization of the transition state (b), nonpolar solvents, such as benzene, allow only trans-alkene formation.

The first attempts were focused on the arsa-Wittig condensation between benzyltriphenyl-arsonium ylide and 1-azulenecarbaldehydes. We chose to start from iodide as ylide precursor salt because it seems that the benzyl iodide reacts faster than other halides with $\mathrm{Ph}_{3} \mathrm{As}$ [20]. Thus, a mixture of triphenylarsine, benzyl chloride and $\mathrm{NaI}$ was refluxed in acetonitrile for 4 hours yielding the corresponding benzyltriphenylarsonium iodides, 3H (Scheme 3) [15].

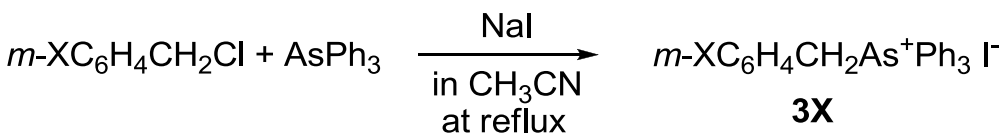

$$
\begin{aligned}
& \mathbf{X}=\mathrm{a}: \mathrm{H} ; \mathrm{b}: \mathrm{OMe} ; \mathrm{c}: \mathrm{Me} ; \mathrm{d}: \mathrm{CF}_{3} ; \mathrm{e}: \mathrm{F} ; \mathrm{f}: \mathrm{NO}_{2}
\end{aligned}
$$

Scheme 3. Synthesis of benzyltriphenylarsonium iodides

The reaction of benzyltriphenylarsonium ylide, obtained from iodide $\mathbf{3 H}$, with 1-azulenecarbaldehyde, carried as described in the literature (alkoxides in alcohol or $\mathrm{NaNH}_{2}$ in benzene), failed and no desired product could be separated and identified. Repeating the reaction without base, in chloroform, it took place in poor aldehyde conversion. However, in the mass spectrum the molecular ions belonging to 1-styrylazulene and to the corresponding epoxide are well represented.

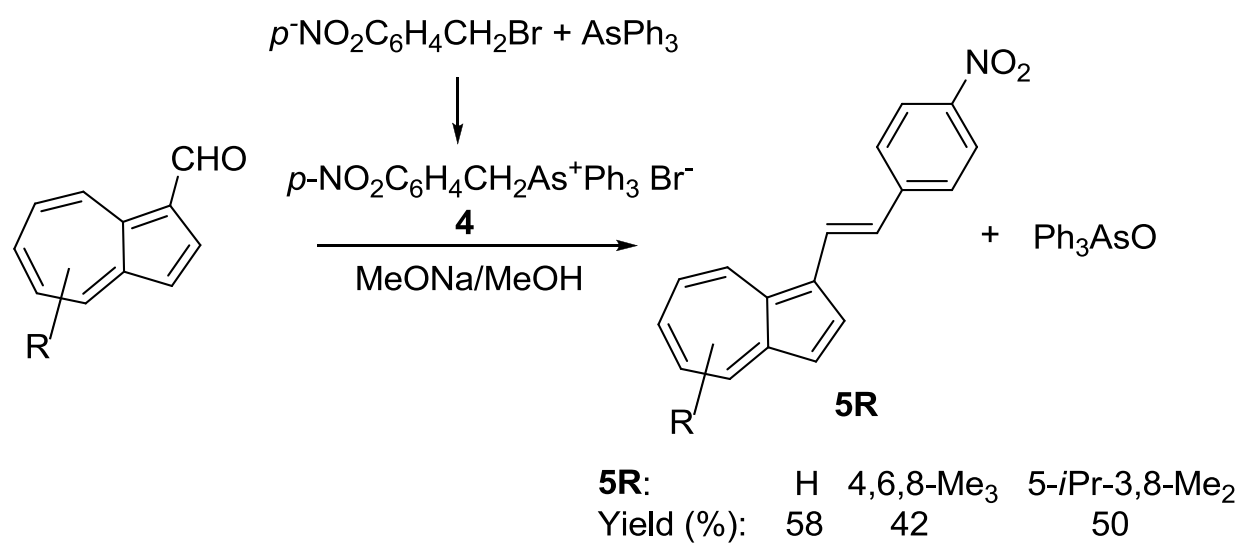

Scheme 4. Arsa-Wittig synthesis of 1-(4-nitrostyryl)azulenes

The contradictions in the literature regarding the behavior of ylides in the condensation with carbonylic compounds prompted us to study the reactions between ylide coming from 4-nitrobenzyltriphenylarsonium bromides, $\mathbf{4}$, and several 1-azulenecarbaldehydes (Scheme 4). The ylide, generated in methanol in the presence of sodium methoxide, reacts with 1-azulenecarbaldehydes following the protocol described by Kendurkar and Tewari [19] for this ylide with other aromatic aldehydes. The obtained $E$ isomer $\mathbf{5 R}$ was detected by mass and NMR spectroscopy. However, in the mass spectra of crude reaction mixtures the molecular ions belonging to epoxy derivative are present. Their low stability probably causes their destruction during separation. Unfortunately, despite the good results 
obtained by these authors using for the arsa-Wittig reaction ylides possessing meta- and orthosubstituted benzyl, [14] our attempts failed, possible due to the lower reactivity of 1-azulenecarbaldehyde or lower stability of the products. However, in this aim a series of 3-substituted triphenylarsonium iodides, $\mathbf{3 X}$, were obtained and characterized (Scheme 3).

It should be noted that the use of sodium amide in benzene, described in the literature for generation of ylides and condensation with aldehydes [22] lead to a reaction mixture contained mainly tars and very small amounts of oxygenated azulene compounds.

\section{NMR and UV-Vis spectra NMR spectra}

The first observation refers to the influence of $\mathrm{P}$ and As on the proton chemical shift of methylene group in benzyltriphenylphosphonium and corresponding arsonium salts. Phosphorus seems to slightly deshielded these protons being more electronegative than arsonium. While in the case of the $\mathrm{P}$, a coupling constant $\mathrm{P}-\mathrm{H}$ about $15.5 \mathrm{~Hz}$ is observed splitting $\mathrm{CH}_{2}$ signal into a doublet, the signal for As is present as singlet. The $\mathrm{CH}_{2}$ group is also influenced by the nature and position of substituent at neighboring phenyl. Thus, $\mathrm{CH}_{2}$ chemical shifts increases with the electron withdrawing effect of the substituent in the meta position of arsonium salts, 3X, from $5.18 \mathrm{ppm}$ for $\mathrm{X}=\mathrm{OMe}$ to $5.34 \mathrm{ppm}$ for $\mathrm{X}$ $=\mathrm{CF}_{3}$ and $\mathrm{NO}_{2}$. For para nitro derivative, 4, in which the inductive and electromeric effects are cumulative, the highest value of $5.99 \mathrm{ppm}$ is observed. This difference is in agreement with the reactivity of para nitrobenzyltriphenylarsonium ylide, the only ylide that reacts with low reactive 1azulenecarbaldehydes. It is interesting that the protons belonging to benzyl phenyl are slightly shielded for arsenic ylide while the phenyl protons are slightly shielded for phosphorus one.

Interesting results were observed for chemical shifts of protons at double bond of 1-styrylazulenes, 2R (Table 2). The discussion concerns mainly the meta substituted series in which the examples are more numerous. The chemical shifts of protons at double bond are influenced more by the molecular stereochemistry and less by the phenyl substituent, which can act from this position only by inductive effect. The stronger molecular conjugation for coplanar $E$ isomer deshielded these protons largely than those of isomer $Z$, where the two aromatic moieties hinder each other, preventing a completely planar structure. Supplementary, some influence exerts the aryls anisotropy current for both protons more for $E$ isomer as compared with $Z$ ones.

Table 2. Chemical shifts of protons at double bond of 1-styrylazulenes with meta substituted phenyl, $\mathbf{2} \mathbf{R}$ ( $\delta$ in in ppm in acetone $\left.-\mathrm{d}_{6}\right)$

\begin{tabular}{|c|c|c|c|c|c|c|}
\hline \multirow{2}{*}{ Isomer } & Substituent at & \multicolumn{5}{|c|}{ Substituent at phenyl } \\
\cline { 2 - 7 } & $\mathrm{C} \underline{H}$ & $\mathrm{NO}_{2}$ & $\mathrm{CF}_{3}$ & $\mathrm{~F}$ & $\mathrm{OMe}$ & $\mathrm{Me}$ \\
\hline \multirow{2}{*}{$Z$} & $\mathrm{Ph}$ & 6.76 & 6.73 & 6.65 & 6.65 & 6.64 \\
& $\mathrm{Az}$ & 7.32 & 7.26 & 7.17 & 7.12 & 7.10 \\
\hline \multirow{2}{*}{$E$} & $\mathrm{Ph}$ & 7.68 & 7.40 & 7.30 & 7.29 & 7.26 \\
\cline { 2 - 7 } & $\mathrm{Az}^{*}$ & 8.18 & 8.11 & 8.01 & 7.93 & 7.90 \\
\hline
\end{tabular}

*Unsubstituted azulen-1-yl.

Next, a comparison will be made regarding the influence on the chemical shift of several protons belonging to 1-(nitrophenylvinyl)azulenes exerted by $\mathrm{NO}_{2}$ position at phenyl as well as by the molecular stereochemistry (Scheme 5). The rule pointed out above for the behavior of vinyl protons of isomer pair $E / Z$ is valid for both the isomer meta and para nitro. Thus, the $E$ isomer deshielded these protons as compared with those of isomer $Z$. It is remarkable, however, that in the case of the para isomer the vinyl protons are shielded as compared with the same protons in meta isomer. This could be explained by the lower double bond character of the vinyl group (Scheme 5) due to the extended conjugation, in which the nitro group fully participates. As can be seen, this effect decreases also the magnetic surrounding anisotropy of vinyl group decreasing the influence on $\mathrm{H} 2$ and $\mathrm{H} 8$. 


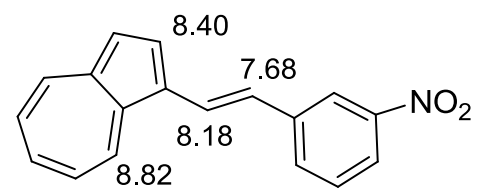

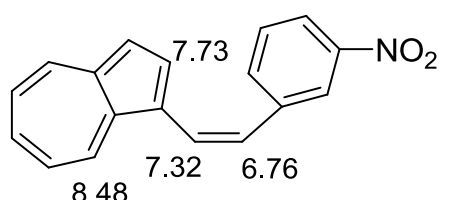

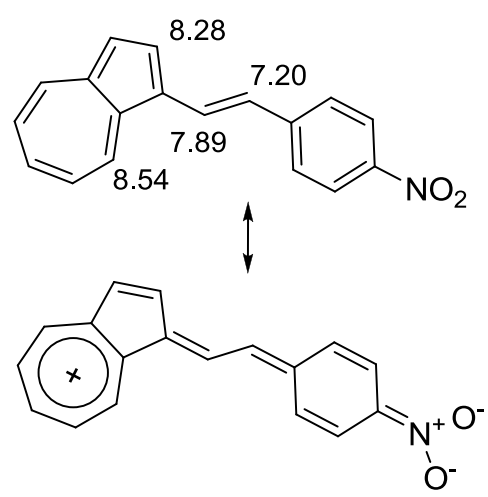<smiles></smiles>

Scheme 5. Chemical shifts for 1-(nitrophenylvinyl)azulenes ( $\delta$ in ppm)

\section{UV-Vis spectra}

Some considerations on the $U V$-Vis spectra of the synthesized 1-styrylazulenes will also refer to the series of more numerous compounds substituted in meta position of phenyl, $\mathbf{2 R}$. The $E$ isomer is more red-shifted than the corresponding $\mathrm{Z}$ isomer due to a better molecular conjugation (Table 3). The substituent does not influence at all the main visible band. At the same time, the para isomers, $\mathbf{5 R}$, are by far more bathochromic, the nitro group being able to function as auxochrome.

Table 3. Main visible band for 1-styrylazulenes (in methanol, $\lambda$ in $\mathrm{nm}$ )

\begin{tabular}{|c|c|c|c|}
\hline Isomer & $\mathrm{NO}_{2}$ & $\mathrm{Me}$ & $\mathrm{OMe}$ \\
\hline Z-meta & 388 & 388 & 387 \\
\hline E-meta & 397 & 396 & 396 \\
\hline Z-para & $436^{*}$ & - & $311^{* *}$ \\
\hline E-para & $454^{*}$ & - & $380^{* *}$ \\
\hline
\end{tabular}

$* \mathrm{In}_{\mathrm{CDCl}}$ [2] **In cyclohexane [17]

\section{Conclusions}

Although arsa-Wittig condensation, but especially Wittig reaction, have a quite old history some aspects related to the reaction mechanism and even the synthesis of some compounds through these procedures continue to interest the researchers. Thus, in this paper some considerations regarding the mechanism of arsa-Wittig condensation have been supplemented with the synthesis of compounds substituted with the nitro to phenyl and various alkyl groups to azulene moiety. These researches were completed by the Wittig synthesis of several unknown meta-substituted 1-styrylazulenes. The obtained products were completely characterized. A look at the behavior of the products in NMR and UV-Vis spectrometry was taken.

\section{References}

1. RAZUS, A. C., BIRZAN, L., ARKIVOC, 2018, IV, p. 1.

2. IFTIME, G., LACROIX, P., NAKATANI, K., RAZUS, A.C., Tetrahedron Lett., 1998, 39, p. 6853.

3. RAZUS, A. C., NITU, C., TECUCEANU, V., CIMPEANU V., Eur. J. Org. Chem., 2003, p. 4601.

4. RAZUS, A. C., BIRZAN, L., TECUCEANU, V., CRISTEA, M., ENACHE, C., Arkivoc, 2008, (xi), p. 210.

5. RAZUS, A. C., BIRZAN, L., TECUCEANU, V., CRISTEA, M., NICOLESCU, A., ENACHE, C., Rev. Roum, Chim., 2007, 52, p. 189. 
6. HUANG, Y. Z., SHEN. Y. C., Adv. Organomet. Chem., 20, 134-150, Academic Press, 1982.

7. LLOYD, D., GOSNEY, I., ORMISTON, R. A., Chem. Soc. Rev., 1987, 16, p. 45.

8. HE, H. S., CHUNG, C. W. Y., BUT, T. Y. S., TOY. P. H., Tetrahedron, 2005, 61, p. 1385.

9. RAECK, C., BERGER S., Eur. J. Org. Chem., 2006, p. 4934.

10. Zeller K.-P. In Methoden der Organischen Chemie; G. Thieme Verlag: Stuttgart, New York, 1985;

Vol. V/2c, p. 127-416.

11. BRIQUET, A. A. S., HANSEN, H.-J., Helv. Chim. Acta, 1994, 77, p. 1921.

12. TAKEKUMA, S., HORI, S., MINEMATSU, T., TAKEKUMA, H., Bull. Chem. Soc. Jpn., 2008, 81, p. 1472.

13. KROHNKE, F., Chem. Ber., 1950, 83, p. 291.

14. KENDURKAR, P. S., TEWARI, R. S., J. Organomet. Chem., 1975, 85, p. 173.

15. BLICKE, F. F., WILLARD, H. H., TARAS, J. T., J. Am. Chem. Soc., 1939, 61, p. 88.

16. MOORHOOF, C. M., Synthetic Commun., 1998, 28, p. 2925.

17. CURRIE Jr., J. O., LABAR, R. A., BREAZEALE, R. D., ANDERSON Jr., A. G., Justus Liebigs Ann. Chem., 1973, p. 166.

18. SCHOLZ, M., KLOTZER, D., MAY, W., MUHLSTADT M., Z. Chem., 1965, p. 237.

19. KENDURKAR, P. S., TEWARI R. S., J. Organomet. Chem., 1973, 60, p. 247.

20. THORSTENSON, T., SONGSTAD, J., Acta Chem. Scand. A, 1976, 30, p. 724.

21. JOHNSON, A. W., SCHUBERT, H., J. Org. Chem., 1970, 35, p. 2678.

22. TEWARI, R. S., CHATURVEDI, S. C., Tetrahedron Lett., 1977, 43, p. 3843.

23. TRIPPETT, S., WALKER., M. A., J. Chem. Soc., C., 1971, p. 1114.

24. BROOS, R., ANTEUNIS, M. J.O., Bull. Soc. Chim. Belg., 1988, 97, p. 271.

25. GOSNEY, I., LILlIE, T. J., LLOYD, D., Angew. Chem. Int. Ed. Engl., 1977, 16, p. 487.

$\overline{\text { Manuscript received: } 14.04 .2020}$ 